\title{
Distortion of Quasiregular Mappings and Equivalent Norms on Lipschitz-Type Spaces
}

\author{
Miodrag Mateljević \\ Faculty of Mathematics, University of Belgrade, Studentski Trg 16, 11000 Belgrade, Serbia \\ Correspondence should be addressed to Miodrag Mateljević; miodrag@matf.bg.ac.rs
}

Received 24 January 2014; Accepted 15 May 2014; Published 21 October 2014

Academic Editor: Ljubomir B. Ćirić

Copyright ( 2014 Miodrag Mateljević. This is an open access article distributed under the Creative Commons Attribution License, which permits unrestricted use, distribution, and reproduction in any medium, provided the original work is properly cited.

\begin{abstract}
We prove a quasiconformal analogue of Koebe's theorem related to the average Jacobian and use a normal family argument here to prove a quasiregular analogue of this result in certain domains in $n$-dimensional space. As an application, we establish that Lipschitztype properties are inherited by a quasiregular function from its modulo. We also prove some results of Hardy-Littlewood type for Lipschitz-type spaces in several dimensions, give the characterization of Lipschitz-type spaces for quasiregular mappings by the average Jacobian, and give a short review of the subject.
\end{abstract}

\section{Introduction}

The Koebe distortion theorem gives a series of bounds for a univalent function and its derivative. A result of Hardy and Littlewood relates Hölder continuity of analytic functions in the unit disk with a bound on the derivative (we refer to this result shortly as HL-result).

Astala and Gehring [1] observed that for certain distortion property of quasiconformal mappings the function $a_{f}$, defined in Section 2, plays analogous role as $\left|f^{\prime}\right|$ when $n=2$ and $f$ is conformal, and they establish quasiconformal version of the well-known result due to Koebe, cited here as Lemma 4, and Hardy-Littlewood, cited here as Lemma 5.

In Section 2, we give a short proof of Lemma 4, using a version with the average Jacobian $J_{f}$ instead of $a_{f}$, and we also characterize bi-Lipschitz mappings with respect to quasihyperbolic metrics by Jacobian and the average Jacobian; see Theorems 8, 9, and 10 and Proposition 13. Gehring and Martio [2] extended HL-result to the class of uniform domains and characterized the domains $D$ with the property that functions which satisfy a local Lipschitz condition in $D$ for some $\alpha$ always satisfy the corresponding global condition there.

The main result of the Nolder paper [3] generalizes a quasiconformal version of a theorem, due to Astala and
Gehring [4, Theorems 1.9 and 3.17] (stated here as Lemma 5) to a quasiregular version (Lemma 33) involving a somewhat larger class of moduli of continuity than $t^{\alpha}, 0<\alpha<1$.

In the paper [5] several properties of a domain which satisfies the Hardy-Littlewood property with the inner length metric are given and also some results on the Hölder continuity are obtained.

The fact that Lipschitz-type properties are sometimes inherited by an analytic function from its modulus was first detected in [6]. Later this property was considered for different classes of functions and we will call shortly results of this type Dyk-type results. Theorem 22 yields a simple approach to Dyk-type result (the part (ii.1); see also [7]) and estimate of the average Jacobian for quasiconformal mappings in space. The characterization of Lipschitz-type spaces for quasiconformal mappings by the average Jacobian is established in Theorem 23 in space case and Theorem 24 yields Dyk-type result for quasiregular mappings in planar case.

In Section 4, we establish quasiregular versions of the well-known result due to Koebe, Theorem 39 here, and use this result to obtain an extension of Dyakonov's theorem for quasiregular mappings in space (without Dyakonov's hypothesis that it is a quasiregular local homeomorphism), Theorem 40. The characterization of Lipschitz-type spaces 
for quasiregular mappings by the average Jacobian is also established in Theorem 40 .

By $\mathbb{R}^{n}=\left\{x=\left(x_{1}, \ldots, x_{n}\right): x_{1}, \ldots, x_{n} \in \mathbb{R}\right\}$ denote the real vector space of dimension $n$. For a domain $D$ in $\mathbb{R}^{n}$ with nonempty boundary, we define the distance function $d=d(D)=\operatorname{dist}(D)$ by $d(x)=d(x ; \partial D)=\operatorname{dist}(D)(x)=$ $\inf \{|x-y|: y \in \partial D\}$, and if $f$ maps $D$ onto $D^{\prime} \subset \mathbb{R}^{n}$, in some settings, it is convenient to use short notation $d_{*}=d_{f}(x)$ for $d\left(f(x) ; \partial D^{\prime}\right)$. It is clear that $d(x)=\operatorname{dist}\left(x, D^{c}\right)$, where $D^{c}$ is the complement of $D$ in $\mathbb{R}^{n}$.

Let $G$ be an open set in $\mathbb{R}^{n}$. A mapping $f: G \rightarrow \mathbb{R}^{m}$ is differentiable at $x \in G$ if there is a linear mapping $f^{\prime}(x)$ : $\mathbb{R}^{n} \rightarrow \mathbb{R}^{n}$, called the derivative of $f$ at $x$, such that

$$
f(x+h)-f(x)=f^{\prime}(x) h+|h| \varepsilon(x, h),
$$

where $\varepsilon(x, h) \rightarrow 0$ as $h \rightarrow 0$. For a vector-valued function $f: G \rightarrow \mathbb{R}^{n}$, where $G \subset \mathbb{R}^{n}$ is a domain, we define

$$
\left|f^{\prime}(x)\right|=\max _{|h|=1}\left|f^{\prime}(x) h\right|, \quad l\left(f^{\prime}(x)\right)=\min _{|h|=1}\left|f^{\prime}(x) h\right|,
$$

when $f$ is differentiable at $x \in G$.

In Section 3, we review some results from [7, 8]. For example, in [7] under some conditions concerning a majorant $\omega$, we showed the following.

Let $f \in C^{1}\left(D, \mathbb{R}^{n}\right)$ and let $\omega$ be a continuous majorant such that $\omega_{*}(t)=\omega(t) / t$ is nonincreasing for $t>0$.

Assume $f$ satisfies the following property (which we call Hardy-Littlewood ( $c, \omega)$-property):

$$
\begin{array}{r}
(\mathrm{HL}(c, \omega)) \quad d(x)\left|f^{\prime}(x)\right| \leq c \omega(d(x)), \\
x \in D, \quad \text { where } d(x)=\operatorname{dist}\left(x, D^{c}\right) .
\end{array}
$$

Then

$$
(\operatorname{loc} \Lambda) \quad f \in \operatorname{loc} \Lambda_{\omega}(G) .
$$

If, in addition, $f$ is harmonic in $D$ or, more generally, $f \in O C^{2}(D)$, then $(\operatorname{HL}(c, \omega))$ is equivalent to $(\operatorname{loc} \Lambda)$. If $D$ is a $\Lambda_{\omega}$-extension domain, then $(\operatorname{HL}(c, \omega))$ is equivalent to $f \in \Lambda_{\omega}(D)$.

In Section 3, we also consider Lipschitz-type spaces of pluriharmonic mappings and extend some results from [9].

In Appendices A and B we discuss briefly distortion of harmonic qc maps, background of the subject, and basic property of qr mappings, respectively. For more details on related qr mappings we refer the interested reader to [10].

\section{Quasiconformal Analogue of Koebe's Theorem and Applications}

Throughout the paper we denote by $\Omega, G$, and $D$ open subset of $\mathbb{R}^{n}, n \geq 1$.

Let $B^{n}(x, r)=\left\{z \in \mathbb{R}^{n}:|z-x|<r\right\}, S^{n-1}(x, r)=\partial B^{n}(x, r)$ (abbreviated $S(x, r)$ ) and let $\mathbb{B}^{n}, S^{n-1}$ stand for the unit ball and the unit sphere in $\mathbb{R}^{n}$, respectively. Sometimes we write $\mathbb{D}$ and $\mathbb{T}$ instead of $\mathbb{B}^{2}$ and $\partial \mathbb{D}$, respectively. For a domain $G \subset$
$\mathbb{R}^{n}$ let $\rho: G \rightarrow[0, \infty)$ be a continuous function. We say that $\rho$ is a weight function or a metric density if, for every locally rectifiable curve $\gamma$ in $G$, the integral

$$
l_{\rho}(\gamma)=\int_{\gamma} \rho(x) d s
$$

exists. In this case we call $l_{\rho}(\gamma)$ the $\rho$-length of $\gamma$. A metric density defines a metric $d_{\rho}: G \times G \rightarrow[0, \infty)$ as follows. For $a, b \in G$, let

$$
d_{\rho}(a, b)=\inf _{\gamma} l_{\rho}(\gamma)
$$

where the infimum is taken over all locally rectifiable curves in $G$ joining $a$ and $b$.

For the modern mapping theory, which also considers dimensions $n \geq 3$, we do not have a Riemann mapping theorem and therefore it is natural to look for counterparts of the hyperbolic metric. So-called hyperbolic type metrics have been the subject of many recent papers. Perhaps the most important metrics of these metrics are the quasihyperbolic metric $\kappa_{G}$ and the distance ratio metric $j_{G}$ of a domain $G \subset \mathbb{R}^{n}$ (see $[11,12])$. The quasihyperbolic metric $\kappa=\kappa_{G}$ of $G$ is a particular case of the metric $d_{\rho}$ when $\rho(x)=1 / d(x, \partial G)$ (see $[11,12])$.

Given a subset $E$ of $\mathbb{C}^{n}$ or $\mathbb{R}^{n}$, a function $f: E \rightarrow \mathbb{C}$ (or, more generally, a mapping $f$ from $E$ into $\mathbb{C}^{m}$ or $\mathbb{R}^{m}$ ) is said to belong to the Lipschitz space $\Lambda_{\omega}(E)$ if there is a constant $L=L(f)=L(f ; E)=L(f, \omega ; E)$, which we call Lipschitz constant, such that

$$
|f(x)-f(y)| \leq L \omega(|x-y|)
$$

for all $x, y \in E$. The norm $\|f\|_{\Lambda_{\omega}(E)}$ is defined as the smallest $L$ in (7).

There has been much work on Lipschitz-type properties of quasiconformal mappings. This topic was treated, among other places, in $[1-5,7,13-22]$.

As in most of those papers, we will currently restrict ourselves to the simplest majorants $\omega_{\alpha}(t):=t^{\alpha}(0<\alpha \leq 1)$. The classes $\Lambda_{\omega}(E)$ with $\omega=\omega_{\alpha}$ will be denoted by $\Lambda^{\alpha}$ (or by $\operatorname{Lip}(\alpha ; E)=\operatorname{Lip}(\alpha, L ; E)) . L(f)$ and $\alpha$ are called, respectively, Lipschitz constant and exponent (of $f$ on $E$ ). We say that a domain $G \subset R^{n}$ is uniform if there are constants $a$ and $b$ such that each pair of points $x_{1}, x_{2} \in G$ can be joined by rectifiable $\operatorname{arc} \gamma$ in $G$ for which

$$
\begin{gathered}
l(\gamma) \leq a\left|x_{1}-x_{2}\right|, \\
\min l\left(\gamma_{j}\right) \leq b d(x, \partial G)
\end{gathered}
$$

for each $x \in \gamma$; here $l(\gamma)$ denotes the length of $\gamma$ and $\gamma_{1}, \gamma_{2}$ the components of $\gamma \backslash\{x\}$. We define $C(G)=\max \{a, b\}$. The smallest $C(G)$ for which the previous inequalities hold is called the uniformity constant of $G$ and we denote it by $c^{*}=c^{*}(G)$. Following $[2,17]$, we say that a function $f$ belongs to the local Lipschitz space loc $\Lambda_{\omega}(G)$ if (7) holds, with a fixed $C>0$, whenever $x \in G$ and $|y-x|<(1 / 2) d(x, \partial G)$. We say that $G$ is a $\Lambda_{\omega}$-extension domain if $\Lambda_{\omega}(G)=\operatorname{loc} \Lambda_{\omega}(G)$. In 
particular if $\omega=\omega_{\alpha}$, we say that $G$ is a $\Lambda^{\alpha}$-extension domain; this class includes the uniform domains mentioned above.

Suppose that $\Gamma$ is a curve family in $\overline{\mathbb{R}}^{n}$. We denote by $\mathscr{F}(\Gamma)$ the family whose elements are nonnegative Borel-measurable functions $\rho$ which satisfy the condition $\int_{\gamma} \rho(x)|d x| \geq 1$ for every locally rectifiable curve $\gamma \in \Gamma$, where $d s=|d x|$ denotes the arc length element. For $p \geq 1$, with the notation

$$
A_{p}(\rho)=\int_{\mathbb{R}^{n}}|\rho(x)|^{p} d V(x)
$$

where $d V(x)=d x$ denotes the Euclidean volume element $d x_{1} d x_{2} \cdots d x_{n}$, we define the $p$-modulus of $\Gamma$ by

$$
M_{p}(\Gamma)=\inf \left\{A_{p}(\rho): \rho \in \mathscr{F}(\Gamma)\right\} .
$$

We will denote $M_{n}(\Gamma)$ simply by $M(\Gamma)$ and call it the modulus of $\Gamma$.

Suppose that $f: \Omega \rightarrow \Omega^{*}$ is a homeomorphism. Consider a path family $\Gamma$ in $\Omega$ and its image family $\Gamma^{*}=\{f \circ \gamma$ : $\gamma \in \Gamma\}$. We introduce the quantities

$$
K_{I}(f)=\sup \frac{M\left(\Gamma^{*}\right)}{M(\Gamma)}, \quad K_{O}(f)=\sup \frac{M(\Gamma)}{M\left(\Gamma^{*}\right)},
$$

where the suprema are taken over all path families $\Gamma$ in $\Omega$ such that $M(\Gamma)$ and $M\left(\Gamma^{*}\right)$ are not simultaneously 0 or $\infty$.

Definition 1. Suppose that $f: \Omega \rightarrow \Omega^{*}$ is a homeomorphism; we call $K_{I}(f)$ the inner dilatation and $K_{O}(f)$ the outer dilatation of $f$. The maximal dilatation of $f$ is $K(f)=$ $\max \left\{K_{O}(f), K_{I}(f)\right\}$. If $K(f) \leq K<\infty$, we say $f$ is $K$ quasiconformal (abbreviated qc).

Suppose that $f: \Omega \rightarrow \Omega^{*}$ is a homeomorphism and $x \in \Omega, x \neq \infty$, and $f(x) \neq \infty$.

For each $r>0$ such that $S(x ; r) \subset \Omega$ we set $L(x, f, r)=$ $\max _{|y-x|=r}|f(y)-f(x)|, l(x, f, r)=\min _{|y-x|=r}|f(y)-f(x)|$.

Definition 2. The linear dilatation of $f$ at $x$ is

$$
H(x, f)=\limsup _{r \rightarrow 0} \frac{L(x, f, r)}{l(x, f, r)} .
$$

Theorem 3 (the metric definition of quasiconformality). A homeomorphism $f: \Omega \rightarrow \Omega^{*}$ is qc if and only if $H(x, f)$ is bounded on $\Omega$.

Let $\Omega$ be a domain in $R^{n}$ and let $f: \Omega \rightarrow R^{n}$ be continuous. We say that $f$ is quasiregular (abbreviated qr) if

(1) $f$ belongs to Sobolev space $W_{1, \text { loc }}^{n}(\Omega)$,

(2) there exists $K, 1 \leq K<\infty$, such that

$$
\left|f^{\prime}(x)\right|^{n} \leq K J_{f}(x) \text { a.e. }
$$

The smallest $K$ in (13) is called the outer dilatation $K_{O}(f)$. A qr mapping is a qc if and only if it is a homeomorphism. First we need Gehring's result on the distortion property of qc (see [23, page 383], [24, page 63]).
Gehring's Theorem. For every $K \geq 1$ and $n \geq 2$, there exists $a$ function $\theta_{K}^{n}:(0,1) \rightarrow \mathbb{R}$ with the following properties:

(1) $\theta_{K}^{n}$ is increasing,

(2) $\lim _{r \rightarrow 0} \theta_{K}^{n}(r)=0$,

(3) $\lim _{r \rightarrow 1} \theta_{K}^{n}(r)=\infty$,

(4) Let $\Omega$ and $\Omega^{\prime}$ be proper subdomains of $\mathbb{R}^{n}$ and let $f$ : $\Omega \rightarrow \Omega^{\prime}$ be a K-qc. If $x$ and $y$ are points in $\Omega$ such that $0<|y-x|<d(x, \partial \Omega)$, then

$$
\frac{|f(y)-f(x)|}{d\left(f(x), \partial \Omega^{\prime}\right)} \leq \theta_{K}^{n}\left(\frac{|y-x|}{d(x, \partial \Omega)}\right) .
$$

Introduce the quantity, mentioned in the introduction,

$$
a_{f}(x)=a_{f, G}(x):=\exp \left(\frac{1}{n\left|B_{x}\right|} \int_{B_{x}} \log J_{f}(z) d z\right), \quad x \in G,
$$

associated with a quasiconformal mapping $f: G \rightarrow f(G) \subset$ $\mathbb{R}^{n}$; here $J_{f}$ is the Jacobian of $f$, while $\mathbf{B}_{x}=\mathbf{B}_{x, G}$ stands for the ball $B(x ; d(x, \partial G))$ and $\left|\mathbf{B}_{x}\right|$ for its volume.

Lemma 4 (see [4]). Suppose that $G$ and $G^{\prime}$ are domains in $R^{n}$ : If $f: G \rightarrow G^{\prime}$ is K-quasiconformal, then

$$
\frac{1}{c} \frac{d\left(f(x), \partial G^{\prime}\right)}{d(x, \partial G)} \leq a_{f, G}(x) \leq c \frac{d\left(f(x), \partial G^{\prime}\right)}{d(x, \partial G)}, \quad x \in G,
$$

where $c$ is a constant which depends only on $K$ and $n$.

$$
\text { Set } \alpha_{0}=\alpha_{K}^{n}=K^{1 /(1-n)} \text {. }
$$

Lemma 5 (see [4]). Suppose that $D$ is a uniform domain in $R^{n}$ and that $\alpha$ and $m$ are constants with $0<\alpha \leq 1$ and $m \geq 0$. If $f$ is K-quasiconformal in $D$ with $f(D) \subset R^{n}$ and if

$$
a_{f, D}(x) \leq \operatorname{md}(x, \partial D)^{\alpha-1} \quad \text { for } x \in D
$$

then $f$ has a continuous extension to $\bar{D} \backslash\{\infty\}$ and

$$
\left|f\left(x_{1}\right)-f\left(x_{2}\right)\right| \leq C\left(\left|x_{1}-x_{2}\right|+d\left(x_{1}, \partial D\right)\right)^{\alpha}
$$

for $x_{1}, x_{2} \in \bar{D} \backslash\{\infty\}$, where the constant $C=\widehat{c}(D)$ depends only on $K, n, a, m$ and the uniformity constant $c^{*}=c^{*}(D)$ for $D$. In the case $\alpha \leq \alpha_{0}$, (18) can be replaced by the stronger conclusion that

$$
\left|f\left(x_{1}\right)-f\left(x_{2}\right)\right| \leq C\left(\left|x_{1}-x_{2}\right|\right)^{\alpha} \quad\left(x_{1}, x_{2} \in \bar{D} \backslash\{\infty\}\right) .
$$

Example 6. The mapping $f(x)=|x|^{a-1} x, a=K^{1 /(1-n)}$ is $K$ qc with $a_{f}$ bounded in the unit ball. Hence $f$ satisfies the hypothesis of Lemma 5 with $\alpha=1$. Since $|f(x)-f(0)| \leq$ $|x-0|^{a}=|x|^{a}$, we see that when $K>1$, that is, $a<1=\alpha$, the conclusion (18) in Lemma 5 cannot be replaced by the stronger assertion $\left|f\left(x_{1}\right)-f\left(x_{2}\right)\right| \leq c m\left|x_{1}-x_{2}\right|$. 
Let $\Omega \in \mathbb{R}^{n}$ and $\mathbb{R}^{+}=[0, \infty)$ and $f, g: \Omega \rightarrow \mathbb{R}^{+}$. If there is a positive constant $c$ such that $f(x) \leq c g(x), x \in \Omega$, we write $f \preceq g$ on $\Omega$. If there is a positive constant $c$ such that

$$
\frac{1}{c} g(x) \leq f(x) \leq c g(x), \quad x \in \Omega,
$$

we write $f \approx g$ (or $f=g$ ) on $\Omega$.

Let $G \subset \mathbb{R}^{2}$ be a domain and let $f: G \rightarrow \mathbb{R}^{2}, f=\left(f_{1}, f_{2}\right)$ be a harmonic mapping. This means that $f$ is a map from $G$ into $\mathbb{R}^{2}$ and both $f_{1}$ and $f_{2}$ are harmonic functions, that is, solutions of the two-dimensional Laplace equation

$$
\Delta u=0 .
$$

The above definition of a harmonic mapping extends in a natural way to the case of vector-valued mappings $f: G \rightarrow$ $\mathbb{R}^{n}, f=\left(f_{1}, \ldots, f_{n}\right)$, defined on a domain $G \subset \mathbb{R}^{n}, n \geq 2$.

Let $h$ be a harmonic univalent orientation preserving mapping on a domain $D, D^{\prime}=h(D)$ and $d_{h}(z)=d(h(z)$, $\left.\partial D^{\prime}\right)$. If $h=f+\bar{g}$ has the form, where $f$ and $g$ are analytic, we define $\lambda_{h}(z)=D^{-}(z)=\left|f^{\prime}(z)\right|-\left|g^{\prime}(z)\right|$, and $\Lambda_{h}(z)=$ $D^{+}(z)=\left|f^{\prime}(z)\right|+\left|g^{\prime}(z)\right|$.

2.1. Quasihyperbolic Metrics and the Average Jacobian. For harmonic qc mappings we refer the interested reader to [25$28]$ and references cited therein.

Proposition 7. Suppose $D$ and $D^{\prime}$ are proper domains in $\mathbb{R}^{2}$. If $h: D \rightarrow D^{\prime}$ is $K$-qc and harmonic, then it is bi-Lipschitz with respect to quasihyperbolic metrics on $D$ and $D^{\prime}$.

Results of this type have been known to the participants of Belgrade Complex Analysis seminar; see, for example, $[29,30]$ and Section 2.2 (Proposition 13, Remark 14 and Corollary 16). This version has been proved by Manojlović [31] as an application of Lemma 4. In [8], we refine her approach.

Proof. Let $h=f+\bar{g}$ be local representation on $B_{z}$.

Since $h$ is $K$-qc, then

$$
\left(1-k^{2}\right)\left|f^{\prime}\right|^{2} \leq J_{h} \leq K\left|f^{\prime}\right|^{2}
$$

on $B_{z}$ and since $\log \left|f^{\prime}(\zeta)\right|$ is harmonic,

$$
\log \left|f^{\prime}(z)\right|=\frac{1}{2\left|B_{z}\right|} \int_{B_{z}} \log \left|f^{\prime}(\zeta)\right|^{2} d \xi d \eta .
$$

Hence,

$$
\begin{aligned}
\log a_{h, D}(z) & \leq \frac{1}{2} \log K+\frac{1}{2\left|B_{z}\right|} \int_{B_{z}} \log \left|f^{\prime}(\zeta)\right|^{2} d \xi d \eta \\
& =\log \sqrt{K}\left|f^{\prime}(z)\right| .
\end{aligned}
$$

Hence, $a_{h, D}(z) \leq \sqrt{K}\left|f^{\prime}(z)\right|$ and $\sqrt{1-k}\left|f^{\prime}(z)\right| \leq a_{h, D}(z)$. Using Astala-Gehring result, we get

$$
\Lambda(h, z)=\frac{d\left(h z, \partial D^{\prime}\right)}{d(z, \partial D)}=\lambda(h, z) .
$$

This pointwise result, combined with integration along curves, easily gives

$$
k_{D^{\prime}}\left(h\left(z_{1}\right), h\left(z_{2}\right)\right)=k_{D}\left(z_{1}, z_{2}\right), \quad z_{1}, z_{2} \in D .
$$

Note that we do not use that $\log \left(1 / J_{h}\right)$ is a subharmonic function.

The following follows from the proof of Proposition 7:

(I) $\Lambda(h, z) \approx a_{h, D} \approx \lambda(h, z)$ and $\sqrt[2]{J_{f}} \approx \underline{J}_{f}$; see below for the definition of average jacobian $I_{f}$.

When underlining a symbol (we also use other latexsymbols) we want to emphasize that there is a special meaning of it; for example we denote by $c$ a constant and by $\underline{c}, \widehat{c}, \tilde{c}$ some specific constants.

Our next result concerns the quantity

$$
\underline{E}_{f, G}(x):=\frac{1}{\left|\underline{B}_{x}\right|} \int_{\underline{B}_{x}} J_{f}(z) d z, \quad x \in G,
$$

associated with a quasiconformal mapping $f: G \rightarrow f(G) \subset$ $\mathbb{R}^{n}$; here $J_{f}$ is the Jacobian of $f$, while $\underline{B}_{x}=\underline{B}_{x, G}$ stands for the ball $B(x, d(x, \partial G) / 2)$ and $\left|B_{x}\right|$ for its volume.

Define

$$
\underline{J}_{f}=\underline{J}_{f, G}=\sqrt[n]{\underline{E}_{f, G}} .
$$

Using the distortion property of qc (see [24, page 63]) we give short proof of a quasiconformal analogue of Koebe's theorem (related to Astala and Gehring's results from [4], cited as Lemma 4 here).

Theorem 8. Suppose that $G$ and $G^{\prime}$ are domains in $\mathbb{R}^{n}$ : If $f$ : $G \rightarrow G^{\prime}$ is $K$-quasiconformal, then

$$
\frac{1}{c} \frac{d\left(f(x), \partial G^{\prime}\right)}{d(x, \partial G)} \leq I_{f, G}(x) \leq c \frac{d\left(f(x), \partial G^{\prime}\right)}{d(x, \partial G)}, \quad x \in G,
$$

where $c$ is a constant which depends only on $K$ and $n$.

Proof. By the distortion property of qc (see [23, page 383], [24, page 63]), there are the constants $C_{*}$ and $c_{*}$ which depend on $n$ and $K$ only, such that

$$
B\left(f(x), c_{*} d_{*}\right) \subset f\left(B_{x}\right) \subset B\left(f(x), C_{*} d_{*}\right), \quad x \in G,
$$

where $d_{*}(x):=d(f(x))=d\left(f(x), \partial G^{\prime}\right)$ and $d(x):=d(x$, $\partial G)$. Hence

$$
\left|B\left(f(x), c_{*} d_{*}\right)\right| \leq \int_{B_{x}} J_{f}(z) d z \leq\left|B\left(f(x), C_{*} d_{*}\right)\right|
$$

and therefore we prove Theorem 8 .

We only outline proofs in the rest of this subsection. $\mathbb{R}^{n}$. 
Theorem 9. Suppose $f: \Omega \rightarrow \Omega^{\prime}$ is a $C^{1}$ and the following hold.

(i1) $f$ is c-Lipschitz with respect to quasihyperbolic metrics on $\Omega$ and $\Omega^{\prime}$; then

$$
\text { (I1) } d\left|f^{\prime}\left(x_{0}\right)\right| \leq c d_{*}\left(x_{0}\right) \text { for every } x_{0} \in \Omega \text {. }
$$

(i2) If $f$ is a qc c-quasihyperbolic-isometry, then

(I2) $f$ is a $c^{2}$-qc mapping,

(I3) $\left|f^{\prime}\right| \approx d_{*} / d$.

Proof. Since $\Omega$ and $\Omega^{\prime}$ are different from $\mathbb{R}^{n}$, there are quasihyperbolic metrics on $\Omega$ and $\Omega^{\prime}$. Then for a fixed $x_{0} \in \Omega$, we have

$$
\begin{array}{r}
k\left(x, x_{0}\right)=d\left(x_{0}\right)^{-1}\left|x-x_{0}\right|(1+o(1)), \quad x \longrightarrow x_{0}, \\
k\left(f(x), f\left(x_{0}\right)\right)=\frac{\left|f(x)-f\left(x_{0}\right)\right|}{d_{*}\left(f\left(x_{0}\right)\right)}(1+o(1)), \\
\text { when } x \longrightarrow x_{0} .
\end{array}
$$

Hence, (i1) implies (I1). If $f$ is a $c$-quasihyperbolic-isometry, then $d\left|f^{\prime}\left(x_{0}\right)\right| \leq c d_{*}$ and $d l\left(f^{\prime}\left(x_{0}\right)\right) \geq d_{*} / c$. Hence, (I2) and (I3) follow.

Theorem 10. Suppose $f: \Omega \rightarrow \Omega^{\prime}$ is a $C^{1}$ qc homeomorphism. The following conditions are equivalent:
(a.1) $f$ is bi-Lipschitz with respect to quasihyperbolic metrics on $\Omega$ and $\Omega^{\prime}$,
(b.1) $\sqrt[n]{J_{f}} \approx d_{*} / d$,
(c.1) $\sqrt[n]{J_{f}} \approx a_{f}$,
(d.1) $\sqrt[n]{J_{f}} \approx I_{f}$,

where $d(x)=d(x, \partial \Omega)$ and $d_{*}(x)=d\left(f(x), \partial \Omega^{\prime}\right)$.

Proof. It follows from Theorem 9 that (a) is equivalent to (b) (see, e.g., $[32,33])$.

Theorem 8 states that $J_{-f} \approx d_{*} / d$. By Lemma $4, a_{f} \approx d_{*} / d$ and therefore (b) is equivalent to (c). The rest of the proof is straightforward.

Lemma 11. If $f \in C^{1,1}$ is a $K$-quasiconformal mapping defined in a domain $\Omega \subset \mathbb{R}^{n}(n \geq 3)$, then

$$
J_{f}(x)>0, \quad x \in \Omega
$$

provided that $K<2^{n-1}$. The constant $2^{n-1}$ is sharp.

If $\bar{G} \subset \Omega$, then it is bi-Lipschitz with respect to Euclidean and quasihyperbolic metrics on $G$ and $G^{\prime}=f(G)$.

It is a natural question whether is there an analogy of Theorem 10 if we drop the $C^{1}$ hypothesis.
Suppose $f: \Omega \rightarrow \Omega^{\prime}$ is onto qc mapping. We can consider the following conditions:

(a.2) $f$ is bi-Lipschitz with respect to quasihyperbolic metrics on $\Omega$ and $\Omega^{\prime}$;

(b.2) $\sqrt[n]{J_{f}} \approx d_{*} / d$ a.e. in $\Omega$;

(c.2) $\sqrt[n]{J_{f}} \approx a_{f}$ a.e. in $\Omega$;

(d.2) $\sqrt[n]{J_{f}} \approx \underline{J}_{f}$ a.e. in $\Omega$.

It seems that the above conditions are equivalent, but we did not check details.

If $\Omega$ is a planar domain and $f$ harmonic qc, then we proved that (d.2) holds (see Proposition 18).

2.2. Quasi-Isometry in Planar Case. For a function $h$, we use notations $\partial h=(1 / 2)\left(h_{x}^{\prime}-i h_{y}^{\prime}\right)$ and $\bar{\partial} h=(1 / 2)\left(h_{x}^{\prime}+i h_{y}^{\prime}\right)$; we also use notations $D h$ and $\bar{D} h$ instead of $\partial h$ and $\bar{\partial} h$, respectively, when it seems convenient. Now we give another proof of Proposition 7, using the following.

Proposition 12 (see [29]). Let $h$ be an euclidean harmonic orientation preserving univalent mapping of the unit disc $\mathbb{D}$ into $\mathbb{C}$ such that $h(\mathbb{D})$ contains a disc $B_{R}=B(a ; R)$ and $h(0)=a$. Then

$$
|\partial h(0)| \geq \frac{R}{4} .
$$

If, in addition, $h$ is $K-q c$, then

$$
\lambda_{h}(0) \geq \frac{1-k}{4} R .
$$

For more details, in connection with material considered in this subsection, see also Appendix A, in particular, Proposition A.7.

Let $h=f+\bar{g}$ be a harmonic univalent orientation preserving mapping on the unit disk $\mathbb{D}, \Omega=h(\mathbb{D}), d_{h}(z)=$ $d(h(z), \partial \Omega), \lambda_{h}(z)=D^{-}(z)=\left|f^{\prime}(z)\right|-\left|g^{\prime}(z)\right|$, and $\Lambda_{h}(z)=$ $D^{+}(z)=\left|f^{\prime}(z)\right|+\left|g^{\prime}(z)\right|$. By the harmonic analogue of the Koebe Theorem, then

$$
\frac{1}{16} D^{-}(0) \leq d_{h}(0)
$$

and therefore

$$
\left(1-|z|^{2}\right) \frac{1}{16} D^{-}(z) \leq d_{h}(z) .
$$

If, in addition, $h$ is $K$-qc, then

$$
\left(1-|z|^{2}\right) D^{+}(z) \leq 16 K d_{h}(z) .
$$

Using Proposition 12, we also prove

$$
\left(1-|z|^{2}\right) \lambda_{h}(z) \geq \frac{1-k}{4} d_{h}(z) .
$$

If we summarize the above considerations, we have proved the part (a.3) of the following proposition. 
Proposition 13 (e-qch, hyperbolic distance version). (a.3). Let $h=f+\bar{g}$ be a harmonic univalent orientation preserving $K$-qc mapping on the unit disk $\mathbb{D}, \Omega=h(\mathbb{D})$. Then, for $z \in \mathbb{D}$,

$$
\begin{aligned}
& \left(1-|z|^{2}\right) \Lambda_{h}(z) \leq 16 K d_{h}(z), \\
& \left(1-|z|^{2}\right) \lambda_{h}(z) \geq \frac{1-k}{4} d_{h}(z) .
\end{aligned}
$$

(b.3) If $h$ is a harmonic univalent orientation preserving $K$ qc mapping of domain $D$ onto $D^{\prime}$, then

$$
\begin{aligned}
& d(z) \Lambda_{h}(z) \leq 16 K d_{h}(z), \\
& d(z) \lambda_{h}(z) \geq \frac{1-k}{4} d_{h}(z),
\end{aligned}
$$

and

$$
\begin{aligned}
\frac{1-k}{4} \kappa_{D}\left(z, z^{\prime}\right) & \leq \kappa_{D^{\prime}}\left(h z, h z^{\prime}\right) \\
& \leq 16 K \kappa_{D}\left(z, z^{\prime}\right),
\end{aligned}
$$

where $z, z^{\prime} \in D$.

Remark 14. In particular, we have Proposition 7, but here the proof of Proposition 13 is very simple and it it is not based on Lemma 4.

Proof of (b.3). Applying (a.3) to the disk $B_{z}, z \in D$, we get (41). It is clear that (41) implies (42).

For a planar hyperbolic domain in $\mathbb{C}$, we denote by $\rho=\rho_{D}$ and $d_{\text {hyp }}=d_{\text {hyp;D }}$ the hyperbolic density and metric of $D$, respectively.

We say that a domain $D \subset \mathbb{C}$ is strongly hyperbolic if it is hyperbolic and diameters of boundary components are uniformly bounded from below by a positive constant.

Example 15. The Poincaré metric of the punctured disk $\mathbb{D}^{\prime}=$ $\{0<|z|<1\}$ is obtained by mapping its universal covering, an infinitely-sheeted disk, on the half plane $\Pi^{-}=\{\operatorname{Re} w<0\}$ by means of $w=\ln z$ (i.e., $z=e^{w}$ ). The metric is

$$
\frac{|d w|}{|\operatorname{Re} w|}=\frac{|d z|}{|z| \ln (1 /|z|)} .
$$

Since a boundary component is the point 0 , the punctured disk is not a strongly hyperbolic domain. Note also that $\rho / d$ and $1 / \ln (1 / d)$ tend to 0 if $z$ tends to 0 . Here $d=d(z)=$ $\operatorname{dist}\left(z, \partial \mathbb{D}^{\prime}\right)$ and $\rho$ is the hyperbolic density of $\mathbb{D}^{\prime}$. Therefore one has the following:

(A.1) for $z \in \mathbb{D}^{\prime}, \kappa_{\mathbb{D}^{\prime}}\left(z, z^{\prime}\right)=\ln (1 / d(z)) d_{\mathrm{hyp}, \mathbb{D}^{\prime}}\left(z, z^{\prime}\right)$ when $z^{\prime} \rightarrow z$.

There is no constant $c$ such that

(A.2) $\kappa_{\mathbb{D}^{\prime}}\left(z, z^{\prime}\right) \leq c \kappa_{\Pi^{-}}\left(w, w^{\prime}\right)$ for every $w, w^{\prime} \in \Pi^{-}$, where $z=e^{w}$ and $z^{\prime}=e^{w^{\prime}}$.

Since $d_{\text {hyp, } \Pi^{-}} \approx \kappa_{\Pi^{-}}$, if (A.2) holds, we conclude that $\kappa_{\mathbb{D}^{\prime}} \approx$ $d_{\text {hyp, } \mathbb{D}^{\prime}}$, which is a contradiction by (A.1).
Let $D$ be a planar hyperbolic domain. Then if $D$ is simply connected,

$$
\frac{1}{2 d} \leq \rho \leq \frac{2}{d} .
$$

For general domain as $z \rightarrow \partial D$,

$$
\frac{1+o(1)}{d \ln (1 / d)} \leq \rho \leq \frac{2}{d} .
$$

Hence $d_{\text {hyp }}\left(z, z^{\prime}\right) \leq 2 \kappa_{D}\left(z, z^{\prime}\right), z, z^{\prime} \in D$.

If $\Omega$ is a strongly hyperbolic domain, then there is a hyperbolic density $\rho$ on the domain $\Omega$, such that $\rho \approx d^{-1}$, where $d(w)=d(w, \partial \Omega)$; see, for example, [34]. Thus $d_{\mathrm{hyp} ; D} \approx$ $\kappa_{D}$. Hence, we find the following.

Corollary 16. Every e-harmonic quasiconformal mapping of the unit disc (more generally of a strongly hyperbolic domain) is a quasi-isometry with respect to hyperbolic distances.

Remark 17. Let $D$ be a hyperbolic domain, let $h$ be e-harmonic quasiconformal mapping of $D$ onto $\Omega$, and let $\phi: \mathbb{D} \rightarrow$ $D$ be a covering and $h^{*}=h \circ \phi$.

(a.4) Suppose that $D$ is simply connected. Thus $\phi$ and $h^{*}$ are one-to-one. Then

$$
\left(1-|z|^{2}\right) \lambda_{h^{*}}(\zeta) \geq \frac{1-k}{4} d_{h}(z)
$$

where $z=\phi(\zeta)$.

Hence, since $\lambda_{h^{*}}(\zeta)=l_{h}(z)\left|\phi^{\prime}(\zeta)\right|$ and $\rho_{\text {hyp;D }}(z)\left|\phi^{\prime}(\zeta)\right|=$ $\rho_{\mathbb{D}}(\zeta)$

$$
\lambda_{h}(z) \geq \frac{1-k}{4} d_{h}(z) \rho_{D}(z)
$$

Using Hall's sharp result, one can also improve the constant in the second inequality in Propositions 13 and 12 (i.e., the constant $1 / 4$ can be replaced by $\tau_{0}$; see below for more details):

$$
\left(1-|z|^{2}\right) \lambda_{h}(z) \geq(1-k) \tau_{0} d_{h}(z),
$$

where $\tau_{0}=3 \sqrt{3} / 2 \pi$.

(b.4) Suppose that $D$ is not a simply connected. Then $h^{*}$ is not one-to-one and we cannot apply the procedure as in (a.4).

(c.4) It seems natural to consider whether there is an analogue in higher dimensions of Proposition 13.

Proposition 18. For every e-harmonic quasiconformal mapping $f$ of the unit disc (more generally of a hyperbolic domain D) the following holds:

(e.2) $\sqrt{J_{f}} \approx d_{*} / d$.

In particular, it is a quasi-isometry with respect to quasihyperbolic distances. 
Proof. For $z \in D$, by the distortion property,

$$
\left|f\left(z_{1}\right)-f(z)\right| \leq c d(f(z)) \quad \text { for every } z_{1} \in \underline{B}_{z} .
$$

Hence, by Schwarz lemma for harmonic maps, $d\left|f^{\prime}(z)\right| \leq$ $c_{1} d(f(z))$. Proposition 12 yields (e) and an application of Proposition 13 gives the proof.

Recall $\underline{B}_{x}$ stands for the ball $B(x ; d(x, \partial G) / 2)$ and $\left|\underline{B}_{x}\right|$ for its volume. If $V$ is a subset of $\mathbb{R}^{n}$ and $u: V \rightarrow \mathbb{R}^{m}$, we define

$$
\begin{gathered}
\operatorname{osc}_{V} u:=\sup \{|u(x)-u(y)|: x, y \in V\}, \\
\omega_{u}(r, x):=\sup \{|u(x)-u(y)|:|x-y|=r\} .
\end{gathered}
$$

Suppose that $G \subset \mathbb{R}^{n}$ and $\underline{B}_{x}=B(x, d(x) / 2)$. Let $O C^{1}(G)=O C^{1}\left(G ; c_{1}\right)$ denote the class of $f \in C^{1}(G)$ such that

$$
d(x)\left|f^{\prime}(x)\right| \leq c_{1} \operatorname{osc}_{\underline{B}_{x}} f
$$

for every $x \in G$.

Proposition 19. Suppose $f: \Omega \rightarrow \Omega^{\prime}$ is a $C^{1}$. Then $f \in O C^{1}(\Omega ; c)$, if and only if $f$ is c-Lipschitz with respect to quasihyperbolic metrics on $B$ and $f(B)$ for every ball $B \subset \Omega$.

2.3. Dyk-Type Results. The characterization of Lipschitz-type spaces for quasiconformal mappings in space and planar quasiregular mappings by the average Jacobian are the main results in this subsection. In particular, using the distortion property of qc mappings we give a short proof of a quasiconformal version of a Dyakonov theorem which states:

Suppose $G$ is a $\Lambda^{\alpha}$-extension domain in $\mathbb{R}^{n}$ and $f$ is a $K$-quasiconformal mapping of $G$ onto $f(G) \subset \mathbb{R}^{n}$. Then $f \in \Lambda^{\alpha}(G)$ if and only if $|f| \in \Lambda^{\alpha}(G)$.

This is Theorem B.3, in Appendix B below. It is convenient to refer to this result as Theorem Dy; see also Theorems 23-24 and Proposition A, Appendix B.

First we give some definitions and auxiliary results. Recall, Dyakonov [15] used the quantity

$$
\underline{E}_{f, G}(x): \frac{1}{\left|\underline{B}_{x}\right|} \int_{\underline{B}_{x}} J_{f}(z) d z, \quad x \in G,
$$

associated with a quasiconformal mapping $f: G \rightarrow f(G) \subset$ $R^{n}$; here $J_{f}$ is the Jacobian of $f$, while $\underline{B}_{x}=\underline{B}_{x, G}$ stands for the ball $B(x ; d(x, \partial G) / 2)$ and $\left|B_{x}\right|$ for its volume.

Define $\alpha_{0}=\alpha_{K}^{n}=K^{1 /(1-n)}, C_{*}=\theta_{K}^{n}(1 / 2), \theta_{K}^{n}\left(c_{*}\right)=1 / 2$, and

$$
\underline{J}_{f, G}=\sqrt[n]{\underline{E}_{f, G}}
$$

For a ball $B=B(x, r) \subset \mathbb{R}^{n}$ and a mapping $f: B \rightarrow \mathbb{R}^{n}$, we define

$$
E_{f}(B ; x):=\frac{1}{|B|} \int_{B} J_{f}(z) d z, \quad x \in G
$$

and $J_{f}^{a v}(B ; x)=\sqrt[n]{E_{f}(B ; x)}$; we also use the notation $J_{f}(B ; x)$ and $J_{f}(x ; r)$ instead of $J_{f}^{a v}(B ; x)$. by

For $x, y \in \mathbb{R}^{n}$, we define the Euclidean inner product $\langle\cdot, \cdot\rangle$

$$
\langle x, y\rangle=x_{1} y_{1}+\cdots+x_{n} y_{n}
$$

By $\mathbb{E}^{n}$ we denote $\mathbb{R}^{n}$ with the Euclidean inner product and call it Euclidean space $n$-space (space of dimension $n$ ). In this paper, for simplicity, we will use also notation $\mathbb{R}^{n}$ for $\mathbb{E}^{n}$. Then the Euclidean length of $x \in \mathbb{R}^{n}$ is defined by

$$
|x|=\langle x, x\rangle^{1 / 2}=\left(\left|x_{1}\right|^{2}+\cdots+\left|x_{n}\right|^{2}\right)^{1 / 2} .
$$

The minimal analytic assumptions necessary for a viable theory appear in the following definition.

Let $\Omega$ be a domain in $\mathbb{R}^{n}$ and let $f: \Omega \rightarrow \mathbb{R}^{n}$ be continuous. We say that $f$ has finite distortion if

(1) $f$ belongs to Sobolev space $W_{1, \text { loc }}^{1}(\Omega)$;

(2) the Jacobian determinant of $f$ is locally integrable and does not change sign in $\Omega$;

(3) there is a measurable function $K_{O}=K_{O}(x) \geq 1$, finite a.e., such that

$$
\left|f^{\prime}(x)\right|^{n} \leq K_{O}(x)\left|J_{f}(x)\right| \text { a.e. }
$$

The assumptions (1), (2), and (3) do not imply that $f \in$ $W_{1, \text { loc }}^{n}(\Omega)$, unless of course $K_{O}$ is a bounded function.

If $K_{O}$ is a bounded function, then $f$ is qr. In this setting, the smallest $K$ in (57) is called the outer dilatation $K_{O}(f)$.

If $f$ is qr, also

$$
J_{f}(x) \leq K^{\prime} l\left(f^{\prime}(x)\right)^{n} \text { a.e. }
$$

for some $K^{\prime}, 1 \leq K^{\prime}<\infty$, where $l\left(f^{\prime}(x)\right)=\inf \left\{\left|f^{\prime}(x) h\right|\right.$ : $|h|=1\}$. The smallest $K^{\prime}$ in (58) is called the inner dilatation $K_{I}(f)$ and $K(f)=\max \left(K_{O}(f), K_{I}(f)\right)$ is called the maximal dilatation of $f$. If $K(f) \leq K, f$ is called $K$-quasiregular.

In a highly significant series of papers published in 19661969 Reshetnyak proved the fundamental properties of $\mathrm{qr}$ mappings and in particular the main theorem concerning topological properties of qr mappings: every nonconstant qr map is discrete and open; cf. [11,35] and references cited there.

Lemma 20 (see Morrey's Lemma, Lemma 6.7.1, [10, page $170])$. Let $f$ be a function of the Sobolev class $W^{1, p}\left(\mathbf{B}, \mathbb{E}^{n}\right)$ in the ball $\mathbf{B}=2 B=B\left(x_{0}, 2 R\right), \underline{B}=B\left(x_{0}, R\right), 1 \leq p<\infty$, such that

$$
D_{p}(f, B)=\left(\frac{1}{|B|} \int_{B}\left|f^{\prime}\right|^{p}\right)^{1 / p} \leq M r^{\gamma-1},
$$

where $0<\gamma \leq 1$, holds for every ball $B=B(a, r) \subset \mathbf{B}$. Then $f$ is Hölder continuous in $\underline{B}$ with exponent $\gamma$, and one has $\mid f(x)-$ $f(y)|\leq C| x-\left.y\right|^{\gamma}$, for all $x, y \in \underline{B}$, where $c=4 M \gamma^{-1} 2^{-\gamma}$. Here and in some places we omit to write the volume element $d x$.

We need a quasiregular version of this Lemma. 
Lemma 21. Let $f: \mathbf{B} \rightarrow \mathbb{R}^{n}$ be a K-quasiregular mapping, such that

$$
J_{f}(a ; r) \leq M r^{\gamma-1},
$$

where $0<\gamma \leq 1$, holds for every ball $B=B(a, r) \subset \mathbf{B}$. Then $f$ is Hölder continuous in $\underline{B}$ with exponent $\gamma$, and one has $\mid f(x)-$ $f(y)|\leq C| x-\left.y\right|^{\gamma}$, for all $x, y \in \underline{B}$, where $C=4 M K^{1 / n} \gamma^{-1} 2^{-\gamma}$.

Proof. By hypothesis, $f$ satisfies (57) and therefore $D_{n}(f, B) \leq$ $K^{1 / n} J_{f}(a ; r)$. An application of Lemma 20 to $p=n$ yields proof.

(a.0) By $\widehat{B}(G)$ denote a family of ball $B=B(x)=B(x, r)$ such that $\mathbf{B}=B(x, 2 r) \subset G$. For $B \in \widehat{B}(G)$, define $d^{\prime}=d_{f, \mathbf{B}}^{\prime}=d\left(f(x), \partial \mathbf{B}^{\prime}\right)$ and $R\left(f(x), \mathbf{B}^{\prime}\right):=c_{*} d^{\prime}$, where $\mathbf{B}^{\prime}=f(\mathbf{B})$.

Define $\omega_{\text {loc }}(f, r)=\omega_{\text {loc }, G}(f, r)=\sup \operatorname{osc}_{B} f$ and $r_{0}(G)=$ sup $r(B)$, where supremum is taken over all balls $B=B(a)=$ $B(a, r)$ such that $B_{1}=B(a, 2 r) \subset G$ and $r(B)$ denotes radius of $B$.

Theorem 22. Let $0<\alpha \leq 1, K \geq 1$. Suppose that (a.5) $G$ is a domain in $\mathbb{R}^{n}$ and $f: G \rightarrow \mathbb{R}^{n}$ is $K$-quasiconformal and $G^{\prime}=f(G)$. Then one has the following.

(i.1) For every $x \in G$, there exists two points $x_{1}, x_{2} \in \underline{B}_{x}$ such that $\left|y_{2}\right|-\left|y_{1}\right| \geq R(x)$, where $y_{k}=f\left(x_{k}\right), k=1,2$, and $R(x):=c_{*} d_{*}(x)$.

(ii.1) $\omega_{\mathrm{loc}, G}(f, r) \leq 2 c_{0} \omega_{\mathrm{loc}, G}(|f|, r), 0 \leq r \leq r_{0}(G)$, where $c_{0}=2 C_{*} / c_{*}$.

(iii.1) If, in addition, one supposes that (b.5) $|f| \in \operatorname{loc} \Lambda^{\alpha}(G)$, then for all balls $B=B(x)=B(x, r)$ such that $B_{1}=$ $B(x, 2 r) \subset G, d_{1} \preceq r^{\alpha}$ and in particular $R(x):=$ $c_{*} d_{*}(x) \leq \operatorname{Ld}(x)^{\alpha}, x \in G$.

(iv.1) There is a constant $\underline{c}$ such that

$$
J_{f}(x ; r) \leq \underline{c} r^{\alpha-1}
$$

for every $x_{0} \in G$ and $B=B(x, r) \subset \underline{B}_{x_{0}}$.

(v.1) If, in addition, one supposes that (c.5) $G$ is a $\Lambda^{\alpha}$ extension domain in $\mathbb{R}^{n}$, then $f \in \operatorname{Lip}\left(\alpha, L_{2} ; G\right)$.

Proof. By the distortion property (30), we will prove the following.

(vi.1) For a ball $B=B(x)=B(x, r)$ such that $B_{1}=B(x$, $2 r) \subset G$ there exist two points $x_{1}, x_{2} \in B(x)$ such that $\left|y_{2}\right|-\left|y_{1}\right| \geq R(x)$, where $y_{k}=f\left(x_{k}\right), k=1,2$.

Let $l$ be line throughout 0 and $f(x)$ which intersects the $S(f(x),(x))$ at points $y_{1}$ and $y_{2}$ and $x_{k}=f^{-1}\left(y_{k}\right), k=1,2$. By the left side of (30), $x_{1}, x_{2} \in B(x)$. We consider two cases:

(a) if $0 \notin B(f(x), R(x))$ and $\left|y_{2}\right| \geq\left|y_{1}\right|$, then $\left|y_{2}\right|-\left|y_{1}\right|=$ $2 R(x)$ and $\left|y_{2}\right|-\left|y_{1}\right|=2 R(x)$;

(b) if $0 \in B(f(x), R(x))$, then, for example, $0 \in\left[y_{1}, f(x)\right]$ and if we choose $x_{1}=x$, we find $\left|y_{2}\right|-|f(x)|=R(x)$ and this yields (vi.1).
Let $x \in G$. An application of (vi.1) to $B=\underline{B}_{x}$ yields (i.1). If $\left|x-x^{\prime}\right| \leq r$, then $c_{*} d^{\prime} \leq\left|y_{2}\right|-\left|y_{1}\right|$ and therefore we have the following:

$$
\left(\text { vi. } 1^{\prime}\right)\left|f(x)-f\left(x^{\prime}\right)\right| \leq C_{*} d^{\prime} \leq c_{0}\left(\left|y_{2}\right|-\left|y_{1}\right|\right) \text {, where } c_{0}=
$$
$C_{*} / c_{*}$

If $z_{1}, z_{2} \in B(x, r)$, then $\left|f\left(z_{1}\right)-f\left(z_{2}\right)\right| \leq\left|f\left(z_{1}\right)-f(x)\right|+$ $\left|f\left(z_{2}\right)-f(x)\right|$. Hence, using (vi. $\left.1^{\prime}\right)$, we find (ii.1). Proof of (iii.1). If the hypothesis (b) holds with multiplicative constant $L$, and $\left|x-x^{\prime}\right|=r$, then $c_{*} d^{\prime} \leq\left|y_{2}\right|-\left|y_{1}\right| \leq 2^{\alpha} L r^{\alpha}$ and therefore $\left|f(x)-f\left(x^{\prime}\right)\right| \leq C_{*} d^{\prime} \leq c_{1} r^{\alpha}$, where $c_{1}=$ $\left(C_{*} / c_{*}\right) 2^{\alpha} L$. Hence $d_{1} \preceq r^{\alpha}$ and therefore, in particular, $R(x):=c_{*} d_{*}(x) \leq L d(x)^{\alpha}, x \in G$.

It is clear, by the hypothesis (b), that there is a fixed constant $L_{1}$ such that (vii.1) $|f|$ belongs to $\operatorname{Lip}\left(\alpha, L_{1} ; B\right)$ for every ball $B=B(x, r)$ such that $B_{1}=B(x, 2 r) \subset G$ and, by (ii.1), we have the following:

(viii.1) $L(f, B) \leq L_{2}$ for a fixed constant $L_{2}$ and $f \in$ loc $\operatorname{Lip}\left(\alpha, L_{2} ; G\right)$.

Hence, since, by the hypothesis (c.5), $G$ is a $L^{\alpha}$-extension domain, we get (v.1).

Proof of (iv.1). Let $B=B(x)=B(x, r)$ be a ball such that $\mathbf{B}=B(x, 2 r) \subset G$. Then $E_{g}(B ; x) \approx d_{1}^{n} / r^{n}, J_{f}(B ; x) \approx d_{1} / r$, and, by (iii.1), $d_{1} \preceq r^{\alpha}$ on $G$. Hence

$$
J_{f}(B ; x) \preceq r^{\alpha-1}
$$

on $G$.

Note that one can also combine (iii.1) and Lemma 33 (for details see proof of Theorem 40 below) to obtain (v.1).

Note that as an immediate corollary of (ii.1) we get a simple proof of Dyakonov results for quasiconformal mappings (without appeal to Lemma 33 or Lemma 21, which is a version of Morrey's Lemma).

We enclose this section by proving Theorems 23 and 24 mentioned in the introduction; in particular, these results give further extensions of Theorem-Dy.

Theorem 23. Let $0<\alpha \leq 1, K \geq 1$, and $n \geq 2$. Suppose $G$ is a $\Lambda^{\alpha}$-extension domain in $\mathbb{R}^{n}$ and $f$ is a $K$-quasiconformal mapping of $G$ onto $f(G) \subset \mathbb{R}^{n}$. The following are equivalent:

(i.2) $f \in \Lambda^{\alpha}(G)$,

(ii.2) $|f| \in \Lambda^{\alpha}(G)$,

(iii.2) there is a constant $c_{3}$ such that

$$
J_{f}(x ; r) \leq c_{3} r^{\alpha-1}
$$

for every $x_{0} \in G$ and $B=B(x, r) \subset \underline{B}_{x_{0}}$. If, in addition, $G$ is a uniform domain and if $\alpha \leq \alpha_{0}=K^{1 /(1-n)}$, then (i.2) and (ii.2) are equivalent to

(iv.2) $|f| \in \Lambda^{\alpha}(G, \partial G)$. 
Proof. Suppose (ii.2) holds, so that $|f|$ is $L$-Lipschitz in $G$. Then (iv.1) shows that (iii.2) holds. By Lemma 21, (iii.2) implies (i.2).

We outline less direct proof that (ii.2) implies (ii.1).

One can show first that (ii.2) implies (18) (or more generally (iv.1); see Theorem 22). Using $A(z)=a+R z$, we conclude that $c^{*}(B(a, R))=c^{*}(\mathbb{B})$ and $\widehat{c}(B)=\widehat{c}$ is a fixed constant (which depends only on $K, n, c_{1}$ ), for all balls $B \subset G$. Lemma 5 tells us that (18) holds, with a fixed constant, for all balls $B \subset G$ and all pairs of points $x_{1}, x_{2} \in B$. Further, we pick two points $x, y \in G$ with $|x-y|<d(x, \partial G)$ and apply (18) with $D=B(x ;|x-y|)$, letting $x_{1}=x$ and $x_{2}=y$. The resulting inequality

$$
|f(x)-f(y)| \leq \text { const }|x-y|^{\alpha}
$$

shows that $f \in \operatorname{loc} \Lambda^{\alpha}(G)$, and since $G$ is a $\Lambda^{\alpha}$-extension domain, we conclude that $f \in \Lambda^{\alpha}(G)$.

The implication (ii.4) $\Rightarrow$ (i.4) is thus established. The converse is clear. For the proof that (iii.4) implies (i.4) for $\alpha \leq \alpha_{0}$, see [15].

For a ball $B=B(x, r) \subset \mathbb{R}^{n}$ and a mapping $f: B \rightarrow \mathbb{R}^{n}$, we define

$$
E_{f}(B ; x):=\frac{1}{|B|} \int_{B} J_{f}(z) d z, \quad x \in G
$$

and $J_{f}(B ; x)=\sqrt[n]{E_{f}(B ; x)}$.

We use the factorization of planar quasiregular mappings to prove the following.

Theorem 24. Let $0<\alpha \leq 1, K \geq 1$. Suppose $D$ is a $\Lambda^{\alpha}$ extension domain in $\mathbb{C}$ and $f$ is a $K$-quasiregular mapping of $D$ onto $f(D) \subset \mathbb{C}$. The following are equivalent:

(i.3) $f \in \Lambda^{\alpha}(D)$,

(ii.3) $|f| \in \Lambda^{\alpha}(D)$,

(iii.3) there is a constant $\check{c}$ such that

$$
J_{f}(B ; z) \leq \check{c} r^{\alpha-1}(z),
$$

$$
\text { for every } z_{0} \in D \text { and } B=B(z, r) \subset \underline{B}_{z_{0}} .
$$

Proof. Let $D$ and $W$ be domains in $C$ and $f: D \rightarrow W$ qr mapping. Then there is a domain $G$ and analytic function $\phi$ on $G$ such that $f=\phi \circ g$, where $g$ is quasiconformal; see [36, page 247].

Our proof will rely on distortion property of quasiconformal mappings. By the triangle inequality, (i.4) implies (ii.4). Now, we prove that (ii.4) implies (iii.4).

Let $z_{0} \in D, \zeta_{0}=g\left(z_{0}\right), G_{0}=g\left(B_{z_{0}}\right), W_{0}=\phi\left(G_{0}\right), d=$ $d\left(z_{0}\right)=d\left(z_{0}, \partial D\right)$, and $d_{1}=d_{1}\left(\zeta_{0}\right)=d\left(\zeta_{0}, \partial G\right)$. Let $B=$ $B(z, r) \subset B_{z_{0}}$. As in analytic case there is $\zeta_{1} \in g(B)$ such that ||$\phi\left(\zeta_{1}\right)|-| \phi\left(\zeta_{0}\right)|| \geq c d_{1}\left(\zeta_{0}\right)\left|\phi^{\prime}\left(\zeta_{0}\right)\right|$. If $z_{1}=g^{-1}\left(\zeta_{1}\right)$, then ||$\phi\left(\zeta_{1}\right)|-| \phi\left(\zeta_{0}\right)||=|| f\left(z_{1}\right)|-| f\left(z_{0}\right) \|$. Hence, if $|f|$ is $\alpha$-Hölder, then $d_{1}(\zeta)\left|\phi^{\prime}(\zeta)\right| \leq c_{1} r^{\alpha}$ for $\zeta \in G_{0}$. Hence, since

$$
E_{f}(B ; z)=\frac{1}{\left|B_{z}\right|} \int_{B_{z}}\left|\phi^{\prime}(\zeta)\right| J_{g}(z) d x d y,
$$

we find

$$
J_{f, D}\left(z_{0}\right) \leq c_{2} \frac{r^{\alpha}}{d_{1}\left(\zeta_{0}\right)} J_{g, G}\left(z_{0}\right)
$$

and therefore, using $E_{g}(B ; z) \approx d_{1}^{2} / r^{2}$, we get

$$
J_{f}(B ; z) \leq c_{3} r^{\alpha-1} .
$$

Thus we have (iii.3) with $\check{c}=c_{3}$.

Proof of the implication (iii.4) $\Rightarrow$ (i.4).

An application of Lemma 21 (see also a version of AstalaGehring lemma) shows that $f$ is $\alpha$-Hölder on $\underline{B}_{z_{0}}$ with a Lipschitz (multiplicative) constant which depends only on $\alpha$ and it gives the result.

\section{Lipschitz-Type Spaces of Harmonic and Pluriharmonic Mappings}

3.1. Higher Dimensional Version of Schwarz Lemma. Before giving a proof of the higher dimensional version of the Schwarz lemma we first establish notation.

Suppose that $h: \bar{B}^{n}(a, r) \rightarrow \mathbb{R}^{m}$ is a continuous vectorvalued function, harmonic on $B^{n}(a, r)$, and let

$$
M_{a}^{*}=\sup \left\{|h(y)-h(a)|: y \in S^{n-1}(a, r)\right\} .
$$

Let $h=\left(h^{1}, h^{2}, \ldots, h^{m}\right)$. A modification of the estimate in [37, Equation (2.31)] gives

$$
r\left|\nabla h^{k}(a)\right| \leq n M_{a}^{*}, \quad k=1, \ldots, m
$$

We next extend this result to the case of vector-valued functions. See also [38] and [39, Theorem 6.16].

Lemma 25. Suppose that $h: \bar{B}^{n}(a, r) \rightarrow \mathbb{R}^{m}$ is a continuous mapping, harmonic in $B^{n}(a, r)$. Then

$$
r\left|h^{\prime}(a)\right| \leq n M_{a}^{*} .
$$

Proof. Without loss of generality, we may suppose that $a=0$ and $h(0)=0$. Let

$$
K(x, y)=K_{y}(x)=\frac{r^{2}-|x|^{2}}{n \omega_{n} r|x-y|^{n}},
$$

where $\omega_{n}$ is the volume of the unit ball in $\mathbb{R}^{n}$. Hence, as in [8], for $1 \leq j \leq n$, we have

$$
\frac{\partial}{\partial x_{j}} K(0, \xi)=\frac{\xi_{j}}{\omega_{n} r^{n+1}} .
$$

Let $\eta \in S^{n-1}$ be a unit vector and $|\xi|=r$. For given $\xi$, it is convenient to write $K_{\xi}(x)=K(x, \xi)$ and consider $K_{\xi}$ as function of $x$.

Then

$$
K_{\xi}^{\prime}(0) \eta=\frac{1}{\omega_{n} r^{n+1}}(\xi, \eta) .
$$


Since $|(\xi, \eta)| \leq|\xi||\eta|=r$, we see that

$$
\left|K_{\xi}^{\prime}(0) \eta\right| \leq \frac{1}{\omega_{n} r^{n}}, \quad \text { and therefore }\left|\nabla K_{\xi}(0)\right| \leq \frac{1}{\omega_{n} r^{n}}
$$

This last inequality yields

$$
\begin{aligned}
\left|h^{\prime}(0)(\eta)\right| & \leq \int_{S^{n-1}(a, r)}\left|\nabla K^{y}(0)\right||h(y)| d \sigma(y) \\
& \leq \frac{M_{0}^{*} n \omega_{n} r^{n-1}}{\omega_{n} r^{n}}=\frac{M_{0}^{*} n}{r},
\end{aligned}
$$

where $d \sigma(y)$ is the surface element on the sphere, and the proof is complete.

Let $\mathbb{C}^{n}=\left\{z=\left(z_{1}, \ldots, z_{n}\right): z_{1}, \ldots, z_{n} \in \mathbb{C}\right\}$ denote the complex vector space of dimension $n$. For $a=\left(a_{1}, \ldots, a_{n}\right) \in$ $\mathbb{C}^{n}$, we define the Euclidean inner product $\langle\cdot, \cdot\rangle$ by

$$
\langle z, a\rangle=z_{1} \bar{a}_{1}+\cdots+z_{n} \bar{a}_{n}
$$

where $\bar{a}_{k}(k \in\{1, \ldots, n\})$ denotes the complex conjugate of $a_{k}$. Then the Euclidean length of $z$ is defined by

$$
|z|=\langle z, z\rangle^{1 / 2}=\left(\left|z_{1}\right|^{2}+\cdots+\left|z_{n}\right|^{2}\right)^{1 / 2} \text {. }
$$

Denote a ball in $\mathbb{C}^{n}$ with center $z^{\prime}$ and radius $r>0$ by

$$
\mathbb{B}^{n}\left(z^{\prime}, r\right)=\left\{z \in \mathbb{C}^{n}:\left|z-z^{\prime}\right|<r\right\} .
$$

In particular, $\mathbb{B}^{n}$ denotes the unit ball $\mathbb{B}^{n}(0,1)$ and $\mathbb{S}^{n-1}$ the sphere $\left\{z \in \mathbb{C}^{n}:|z|=1\right\}$. Set $\mathbb{D}=\mathbb{B}^{1}$, the open unit disk in $\mathbb{C}$, and let $T=\mathbb{S}^{0}$ be the unit circle in $\mathbb{C}$.

A continuous complex-valued function $f$ defined in a domain $\Omega \subset \mathbb{C}^{n}$ is said to be pluriharmonic if, for fixed $z \in \Omega$ and $\theta \in \mathbb{S}^{n-1}$, the function $f(z+\theta \zeta)$ is harmonic in $\left\{\zeta \in \mathbb{C}:|\theta \zeta-z|<d_{\Omega}(z)\right\}$, where $d_{\Omega}(z)$ denotes the distance from $z$ to the boundary $\partial \Omega$ of $\Omega$. It is easy to verify that the real part of any holomorphic function is pluriharmonic; $\mathrm{cf}$. [40].

Let $\omega:[0,+\infty) \rightarrow[0,+\infty)$ with $\omega(0)=0$ be a continuous function. We say that $\omega$ is a majorant if

(1) $\omega(t)$ is increasing,

(2) $\omega(t) / t$ is nonincreasing for $t>0$.

If, in addition, there is a constant $C>0$ depending only on $\omega$ such that

$$
\begin{gathered}
\int_{0}^{\delta} \frac{\omega(t)}{t} d t \leq C \omega(\delta), \quad 0<\delta<\delta_{0} \\
\delta \int_{\delta}^{\infty} \frac{\omega(t)}{t^{2}} d t \leq C \omega(\delta), \quad 0<\delta<\delta_{0}
\end{gathered}
$$

for some $\delta_{0}$, then we say that $\omega$ is a regular majorant. A majorant is called fast (resp., slow) if condition (81) (resp., (82)) is fulfilled.

Given a majorant $\omega$, we define $\Lambda_{\omega}(\Omega)\left(\right.$ resp., $\left.\Lambda_{\omega}(\partial \Omega)\right)$ to be the Lipschitz-type space consisting of all complex-valued functions $f$ for which there exists a constant $C$ such that, for all $z$ and $w \in \Omega$ (resp., $z$ and $w \in \partial \Omega$ ),

$$
|f(z)-f(w)| \leq C \omega(|z-w|) .
$$

Using Lemma 25, one can prove the following.

Proposition 26. Let $\omega$ be a regular majorant and let $f$ be harmonic mapping in a simply connected $\Lambda_{\omega}$-extension domain $D \subset R^{n}$. Then $h \in \Lambda_{\omega}(D)$ if and only if $|\nabla f| \leq C(\omega(1-|z|) /(1-$ $|z|))$.

It is easy to verify that the real part of any holomorphic function is pluriharmonic. It is interesting that the converse is true in simply connected domains.

Lemma 27. (i) Let $u$ be pluriharmonic in $B_{0}=B(a ; r)$. Then there is an analytic function $f$ in $B$ such that $f=u+i v$.

(ii) Let $\Omega$ be simply connected and $u$ be pluriharmonic in $\Omega$. Then there is analytic function $f$ in $\Omega$ such that $f=u+i v$.

Proof. (i) Let $B_{0}=B(a ; r) \subset \Omega, P_{k}=-u_{y_{k}}$, and $Q=u_{x_{k}}$; define form

$$
\omega_{k}=P_{k} d x_{k}+Q_{k} d y_{k}=-u_{y_{k}} d x_{k}+u_{x_{k}} d y_{k},
$$

$\omega=\sum_{k=1}^{n} \omega_{k}$ and $v(x, y)=\int_{\left(x_{0}, y_{0}\right)}^{(x, y)} \omega$. Then (i) holds for $f_{0}=$ $u+i v$, which is analytic on $B_{0}$.

(ii) If $z \in \Omega$, there is a chain $C=\left(B_{0}, B_{1}, \ldots, B_{n}\right)$ in $\Omega$ such that $z$ is center of $B_{n}$ and, by the lemma, there is analytic chain $\left(B_{k}, f_{k}\right), k=0, \ldots, n$. We define $f(z)=f_{n}(z)$. As in in the proof of monodromy theorem in one complex variable, one can show that this definition does not depend of chains $C$ and that $f=u+i v$ in $\Omega$.

The following three theorems in [9] are a generalization of the corresponding one in [15].

Theorem 28. Let $\omega$ be a fast majorant, and let $f=h+\bar{g}$ be a pluriharmonic mapping in a simply connected $\Lambda_{\omega}$-extension domain $\Omega$. Then the following are equivalent:

(1) $f \in \Lambda_{\omega}(\Omega)$;

(2) $h \in \Lambda_{\omega}(\Omega)$ and $g \in \Lambda_{\omega}(\Omega)$;

(3) $|h| \in \Lambda_{\omega}(\Omega)$ and $|g| \in \Lambda_{\omega}(\Omega)$;

(4) $|h| \in \Lambda_{\omega}(\Omega, \partial \Omega)$ and $|g| \in \Lambda_{\omega}(\Omega, \partial \Omega)$,

where $\Lambda_{\omega}(\Omega, \partial \Omega)$ denotes the class of all continuous functions $f$ on $\Omega \cup \partial \Omega$ which satisfy (83) with some positive constant $C$, whenever $z \in \Omega$ and $w \in \partial \Omega$.

Define $D f=\left(D_{1} f, \ldots, D_{n} f\right)$ and $\bar{D} f=\left(\bar{D}_{1} f, \ldots, \bar{D}_{n} f\right)$, where $z_{k}=x_{k}+i y_{k}, D_{k} f=(1 / 2)\left(\partial_{x_{k}} f-i \partial_{y_{k}} f\right)$ and $\bar{D}_{k} f=$ $(1 / 2)\left(\partial_{x_{k}} f+i \partial_{y_{k}} f\right), k=1,2, \ldots, n$.

Theorem 29. Let $\Omega \subset \mathbb{C}^{n}$ be a domain and $f$ analytic in $\Omega$. Then

$$
d_{\Omega}(z)|\nabla f(z)| \leq 4 \omega_{|f|}\left(d_{\Omega}(z)\right)
$$

If $|f| \in \Lambda_{\omega}(\Omega)$, then $d_{\Omega}(z)|\nabla f(z)| \leq 4 C \omega\left(d_{\Omega}(z)\right)$. 
If $f=h+\bar{g}$ is a pluriharmonic mapping, where $g$ and $h$ are analytic in $\Omega$, then $\left|g^{\prime}\right| \leq\left|f^{\prime}\right|$ and $\left|h^{\prime}\right| \leq\left|f^{\prime}\right|$. In particular, $\left|D_{i} g\right|,\left|D_{i} h\right| \leq\left|f^{\prime}\right| \leq|D g|+|D h|$.

Proof. Using a version of Koebe theorem for analytic functions (we can also use Bloch theorem), we outline a proof. Let $z \in \Omega, a=\left(a_{1}, \ldots, a_{n}\right) \in \mathbb{C}^{n},|a|=1, F(\lambda)=F(\lambda, a)=$ $f(z+\lambda a), \lambda \in B\left(0, d_{\Omega}(z) / 2\right)$, and $u=|F|$.

By the version of Koebe theorem for analytic functions, for every line $L$ which contains $w=f(z)$, there are points $w_{1}, w_{2} \in L$ such that $d_{\Omega}(z)\left|F^{\prime}(0)\right| \leq 4\left|w_{k}-w\right|, k=1,2$, and $w \in\left[w_{1}, w_{2}\right]$. Hence $d_{\Omega}(z)\left|F^{\prime}(0)\right| \leq 4 \omega_{u}\left(d_{\Omega}(z)\right)$ and $d_{\Omega}(z)\left|F^{\prime}(0)\right| \leq 4 \omega_{|f|}\left(d_{\Omega}(z)\right)$.

Define $f^{\prime}(z)=\left(f_{z_{1}}^{\prime}(z), \ldots f_{z_{n}}^{\prime}(z)\right)$. Since $F^{\prime}(0)=$ $\sum_{k=1}^{n} D_{k} f(z) a_{k}=\left(f^{\prime}(z), \bar{a}\right)$, we find $|\nabla f(z)|=\left|f^{\prime}(z)\right|$, where $\bar{a}=\left(\overline{a_{1}}, \ldots, \overline{a_{n}}\right)$. Hence

$$
d_{\Omega}(z)|\nabla f(z)| \leq 4 \omega_{u}\left(d_{\Omega}(z)\right) \leq 4 \omega_{|f|}\left(d_{\Omega}(z)\right) .
$$

Finally if $|f| \in \Lambda_{\omega}(\Omega)$, we have $d_{\Omega}(z)|\nabla f(z)| \leq$ $4 C \omega\left(d_{\Omega}(z)\right)$.

For $B^{n}$ the following result is proved in [9].

Theorem 30. Let $\omega$ be a regular majorant and let $\Omega$ be a simply connected $\Lambda_{\omega}$-extension domain. A function $f$ pluriharmonic in belongs to $\Lambda_{\omega}(\Omega)$ if and only if, for each $i \in\{1,2, \ldots, n\}$, $d_{\Omega}(z)\left|D_{i} f(z)\right| \leq \omega\left(d_{\Omega}(z)\right)$ and $d_{\Omega}(z)\left|\bar{D}_{i} f(z)\right| \leq \omega\left(d_{\Omega}(z)\right)$ for some constant $C$ depending only on $f, \omega, \Omega$, and $n$.

We only outline a proof: let $f=h+\bar{g}$. Note that $D_{i} f=D_{i} h$ and $\bar{D}_{i} f=\overline{D_{i} g}$.

We can also use Proposition 26.

3.2. Lipschitz-Type Spaces. Let $f: G \rightarrow G^{\prime}$ be a $C^{2}$ function and $\underline{B}_{x}=B(x, d(x) / 2)$. We denote by $O C^{2}(G)$ the class of functions which satisfy the following condition:

$$
\sup _{B_{x}} d^{2}(x)|\Delta f(x)| \leq c \operatorname{osc}_{B_{x}} f,
$$

for every $x \in G$.

It was observed in [8] that $O C^{2}(G) \subset O C^{1}(G)$. In [7], we proved the following results.

Theorem 31. Suppose that

(a1) $D$ is a $\Lambda^{\alpha}$-extension domain in $\mathbb{R}^{n}, 0<\alpha \leq 1$, and $f$ is continuous on $\bar{D}$ which is a $K$-quasiconformal mapping of $D$ onto $G=f(D) \subset \mathbb{R}^{n}$;

(a2) $\partial D$ is connected;

(a3) $f$ is Hölder on $\partial D$ with exponent $\alpha$;

(a4) $f \in O C^{2}(D)$. Then $f$ is Hölder on $\bar{D}$ with exponent $\alpha$

The proof in [7] is based on Lemmas 3 and 8 in the paper of Martio and Nakki [18]. In the setting of Lemma 8, $d\left|f^{\prime}(y)\right| \leq \widehat{M} d^{\alpha}$. In the setting of Lemma 3 , using the fact that $\partial D$ is connected, we get similar estimate for $d$ small enough.
Theorem 32. Suppose that $D$ is a domain in $\mathbb{R}^{n}, 0<\alpha \leq 1$, and $f$ is harmonic (more generally $O C^{2}(D)$ ) in $D$. Then one has the following:

(i.1) $f \in \operatorname{Lip}(\omega, c, D)$ implies

(ii.1) $\left|f^{\prime}(x)\right| \leq c d(x)^{\alpha-1}, x \in D$.

(ii.1) implies

(iii.1) $f \in \operatorname{loc} \operatorname{Lip}\left(\alpha, L_{1} ; D\right)$.

\section{Theorems of Koebe and Bloch Type for Quasiregular Mappings}

We assume throughout that $G \subset \mathbb{R}^{n}$ is an open connected set whose boundary, $\partial G$, is nonempty. Also $B(x, R)$ is the open ball centered at $x \in G$ with radius $R$. If $B \subset \mathbb{R}^{n}$ is a ball, then $\sigma B, \sigma>0$, denotes the ball with the same center as $B$ and with radius equal to $\sigma$ times that of $B$.

The spherical (chordal) distance between two points $a$, $b \in \overline{\mathbb{R}}^{n}$ is the number

$$
\underline{q}(a, b)=|p(a)-p(b)|,
$$

where $p: \overline{\mathbb{R}}^{n} \rightarrow S\left(e_{n+1} / 2,1 / 2\right)$ is stereographic projection, defined by

$$
p(x)=e_{n+1}+\frac{x-e_{n+1}}{\left|x-e_{n+1}\right|^{2}} .
$$

Explicitly, if $a \neq \infty \neq b$,

$$
\underline{q}(a, b)=|a-b|\left(1+|a|^{2}\right)^{-1 / 2}\left(1+|b|^{2}\right)^{-1 / 2} .
$$

When $f: G \rightarrow \mathbb{R}^{n}$ is differentiable, we denote its Jacobi matrix by $D f$ or $f^{\prime}$ and the norm of the Jacobi matrix as a linear transformation by $\left|f^{\prime}\right|$. When $D f$ exists a.e. we denote the local Dirichlet integral of $f$ at $x \in G$ by $\underline{D}_{f}(x)=$ $\underline{D}_{f, G}(x)=\left(1 /|B| \int_{\underline{B}}\left|f^{\prime}\right|^{n}\right)^{1 / n}$, where $\underline{B}=\underline{B}_{x}=\underline{B}_{x, G}$. If there is no chance of confusion, we will omit the index $G$. If $\mathbf{B}=\mathbf{B}_{x}$, then $\underline{D}_{f, G}(x)=\underline{D}_{f, \mathbf{B}}(x)$ and if $\mathbb{B}$ is the unit ball, we write $\underline{D}(f)$ instead of $\underline{D}_{f, \mathbb{B}}(0)$.

When the measure is omitted from an integral, as here, integration with respect to $n$-dimensional Lebesgue measure is assumed.

A continuous increasing function $\omega(t):[0, \infty) \rightarrow$ $[0, \infty)$ is a majorant if $\omega(0)=0$ and if $\omega\left(t_{1}+t_{2}\right) \leq \omega\left(t_{1}\right)+\omega\left(t_{2}\right)$ for all $t_{1}, t_{2} \geq 0$.

The main result of the paper [3] generalizes Lemma 5 to a quasiregular version involving a somewhat larger class of moduli of continuity than $t^{\alpha}, 0<\alpha<1$.

Lemma 33 (see [3]). Suppose that $G$ is $\Lambda_{\omega}$-extension domain in $\mathbb{R}^{n}$. If $f$ is K-quasiregular in $G$ with $f(G) \subset \mathbb{R}^{n}$ and if

$$
\underline{D}_{f}(x) \leq C_{1} \omega(d(x)) d(x, \partial D)^{-1} \quad \text { for } x \in G,
$$

then $f$ has a continuous extension to $\bar{D} \backslash\{\infty\}$ and

$$
\left|f\left(x_{1}\right)-f\left(x_{2}\right)\right| \leq C_{2} \omega\left(\left|x_{1}-x_{2}\right|+d\left(x_{1}, \partial G\right)\right),
$$


for $x_{1}, x_{2} \in \bar{D} \backslash\{\infty\}$, where the constant $C_{2}$ depends only on $K, n, \omega, C_{1}$, and $G$.

If $G$ is uniform, the constant $C_{2}=\widehat{c}(G)$ depends only on $K, n, C_{1}$ and the uniformity constant $c^{*}=c^{*}(G)$ for $G$.

Conversely if there exists a constant $C_{2}$ such that (92) holds for all $x_{1}, x_{2} \in G$, then (91) holds for all $x \in G$ with $C_{1}$ depending only on $C_{2}, K, n, \omega$, and $G$.

Now suppose that $\omega=\omega_{\alpha}$ and $\alpha \leq \alpha_{0}$.

Also in [3], Nolder, using suitable modification of a theorem of Näkki and Palka [41], shows that (92) can be replaced by the stronger conclusion that

$$
\left|f\left(x_{1}\right)-f\left(x_{2}\right)\right| \leq C\left(\left|x_{1}-x_{2}\right|\right)^{\alpha} \quad\left(x_{1}, x_{2} \in \bar{D} \backslash\{\infty\}\right) .
$$

Remark 34. Simple examples show that the term $d\left(x_{1}, \partial G\right)$ cannot in general be omitted. For example, $f(x)=x|x|^{a-1}$ with $a=K^{1 /(1-n)}$ is $K$-quasiconformal in $B=B(0 ; 1) . \underline{D}_{f}(x)$ is bounded over $x \in B$ yet $f \in \operatorname{Lip}_{a}(B)$; see Example 6 .

If $n=2$, then $\left|f^{\prime}(z)\right| \approx \rho^{a-1}$, where $z=\rho e^{i \theta}$, and if $B_{r}=$ $B(0, r)$, then $\int_{B_{r}}\left|f^{\prime}(z)\right| d x d y=\int_{0}^{r} \int_{0}^{2 \pi} \rho^{2(a-1)} \rho d \rho d \theta \approx r^{2 a}$ and $\underline{D}_{f, B_{r}}(0) \approx r^{a-1}$.

Note that we will show below that if $|f| \in L^{\alpha}(G)$, the conclusion (92) in Lemma 33 can be replaced by the stronger assertion $\left|f\left(x_{1}\right)-f\left(x_{2}\right)\right| \leq c m\left|x_{1}-x_{2}\right|^{\alpha}$.

Lemma 35 (see [3]). If $f=\left(f_{1}, f_{2}, \ldots, f_{n}\right)$ is $K$-quasiregular in $G$ with $f(G) \subset R^{n}$ and if $B$ is a ball with $\sigma B \subset G, \sigma>1$, then there exists a constant $C$, depending only on $n$, such that

$$
\left(\frac{1}{|B|} \int_{B}\left|f^{\prime}\right|^{n}\right)^{1 / n} \leq C K \frac{\sigma}{\sigma-1}\left(\frac{1}{|\sigma B|} \int_{\sigma B}\left|f_{j}-a\right|^{n}\right)^{1 / n}
$$

for all $a \in \mathbb{R}$ and all $j=1,2, \ldots, n$. Here and in some places we omit to write the volume and the surface element.

Lemma 36 (see[42], second version of Koebe theorem for analytic functions). Let $B=B(a ; r)$; let $f$ be holomorphic function on $B, D=f(B), f(a)=b$, and let the unbounded component $D_{\infty}$ of $D$ be not empty, and let $d_{\infty}=d_{\infty}(b)=$ $\operatorname{dist}\left(b, D_{\infty}\right)$. Then (a.1) $r\left|f^{\prime}(a)\right| \leq 4 d_{\infty} ;($ b.1) if, in addition, $D$ is simply connected, then $D$ contains the disk $B(b, \rho)$ of radius $\rho$, where $\rho=\rho_{f}(a)=r\left|f^{\prime}(a)\right| / 4$.

The following result can be considered as a version of this lemma for quasiregular mappings in space.

Theorem 37. Suppose that $f$ is a $K$-quasiregular mapping on the unit ball $\mathbb{B}, f(0)=0$ and $|\underline{D}(f)| \geq 1$.

Then, there exists an absolute constant $\alpha$ such that for every $x \in \mathbb{S}$ there exists a point $y$ on the half-line $\Lambda_{x}=\Lambda(0, x)=$ $\{\rho x: \rho \geq 0\}$, which belongs to $f(\mathbb{B})$, such that $|y| \geq 2 \alpha$.

If $f$ is a K-quasiconformal mapping, then there exists an absolute constant $\rho_{1}$ such that $f(\mathbb{B})$ contains $\mathbb{B}\left(0 ; \rho_{1}\right)$.

For the proof of the theorem, we need also the following result, Theorem 18.8.1 [10].
Lemma 38. For each $K \geq 1$ there is $m=m(n, K)$ with the following property. Let $\epsilon>0$, let $G \subset \overline{\mathbb{R}}^{n}$ be a domain, and let $\widehat{F}$ denote the family of all qr mappings with $\max \left\{K_{O}(x, f)\right.$, $\left.K_{I}(x, f)\right\} \leq K$ and $f: G \rightarrow \mathbb{R}^{n} \backslash\left\{a_{1, f}, a_{2, f}, \ldots, a_{m, f}\right\}$ where $a_{i, f}$ are points in $\overline{\mathbb{R}}^{n}$ such that $\underline{q}\left(a_{i, f}, a_{j, f}\right)>\epsilon, i \neq j$. Then $\widehat{F}$ forms a normal family in $G$.

Now we prove Theorem 37.

Proof. If we suppose that this result is not true then there is a sequence of positive numbers $a_{n}$, which converges to zero, and a sequence of $K$-quasiregular functions $f_{n}$, such that $f_{n}(\mathbb{B})$ does not intersect $\left[a_{n},+\infty\right), n \geq 1$. Next, the functions $g_{n}=f_{n} / a_{n}$ map $\mathbb{B}$ into $G=\mathbb{R}^{n} \backslash[1,+\infty)$ and hence, by Lemma 38 , the sequence $g_{n}$ is equicontinuous and therefore forms normal family. Thus, there is a subsequence, which we denote again by $g_{n}$, which converges uniformly on compact subsets of $\mathbb{B}$ to a quasiregular function $g$. Since $\underline{D}\left(g_{n}\right)$ converges to $\underline{D}(g)$ and $\left|\underline{D}\left(g_{n}\right)\right|=\left|\underline{D}\left(f_{n}\right)\right| / a_{n}$ converges to infinity, we have a contradiction by Lemma 35 .

A path-connected topological space $X$ with a trivial fundamental group $\pi_{1}(X)$ is said to be simply connected. We say that a domain $V$ in $\mathbb{R}^{3}$ is spatially simply connected if the fundamental group $\pi_{2}(V)$ is trivial.

As an application of Theorem 37, we immediately obtain the following result, which we call the Koebe theorem for quasiregular mappings.

Theorem 39 (second version of Koebe theorem for $K$-quasiregular functions). Let $B=B(a ; r)$; let $f$ be $K$-quasiregular function on $B \subset R^{n}, D=f(B), f(a)=b$, and let the unbounded component $D_{\infty}$ of $D^{c}$ be not empty, and let $d_{\infty}=$ $d_{\infty}(b)=\operatorname{dist}\left(b, D_{\infty}\right)$. Then there exists an absolute constant $c$ :

(a.1) $r\left|\underline{D}_{f}(a)\right| \leq \widetilde{c} d_{\infty} ;$

(a.2) if, in addition, $D \subset \mathbb{R}^{3}$ and $D$ is spatially simply connected, then $D$ contains the disk $B(b, \rho)$ of radius $\rho$, where $\rho=\rho_{f}(a)=r\left|\underline{D}_{f}(a)\right| / \widetilde{c}$.

(A.1) Now, using Theorem 39 and Lemma 20, we will establish the characterization of Lipschitz-type spaces for quasiregular mappings by the average Jacobian and in particular an extension of Dyakonov's theorem for quasiregular mappings in space (without Dyakonov's hypothesis that it is a quasiregular local homeomorphism). In particular, our approach is based on the estimate (a.3) below.

Theorem 40 (Theorem-DyMa). Let $0<\alpha \leq 1, K \geq 1$, and $n \geq 2$. Suppose $G$ is a $L^{\alpha}$-extension domain in $\mathbb{R}^{n}$ and $f$ is a $K$-quasiregular mapping of $G$ onto $f(G) \subset \mathbb{R}^{n}$. The following are equivalent:

(i.4) $f \in \Lambda^{\alpha}(G)$,

(ii.4) $|f| \in \Lambda^{\alpha}(G)$,

(iii.4) $\left|\underline{D}_{f}(r, x)\right| \leq c r^{\alpha-1}$ for every ball $B=B(x, r) \subset G$. 
Proof. Suppose that $f$ is a $K$-quasiregular mapping of $G$ onto $f(G) \subset \mathbb{R}^{n}$. We first establish that for every ball $B=B(x, r) \subset$ $G$, one has the following:

(a.3) $r\left|\underline{D}_{f}(r, x)\right| \leq \widetilde{c} \omega_{|f|}(r, x)$, where $\underline{D}_{f}(r, x)=\underline{D}_{f, B}(x)$.

Let $l$ be line throughout 0 and $f(x)$ and denote by $D_{\infty, r}$ the unbounded component of $D^{c}$, where $D=f(B(x, r))$. Then, using a similar procedure as in the proof of Theorem 22, the part (iii.1), one can show that there is $z^{\prime} \in \partial D_{\infty, r} \cap l$ such that $d_{\infty}\left(x^{\prime}\right) \leq\left|z^{\prime}-f(x)\right|=|| z^{\prime}|-| f(x) \|$, where $x^{\prime}=f(x)$.

Take a point $z \in f^{-1}\left(z^{\prime}\right)$. Then $z^{\prime}=f(z)$ and $|z-x|=r$. Since ||$f(z)|-| f(x) \| \leq \omega_{|f|}(x, r)$. Then using Theorem 39, we find (a.3).

Now we suppose (ii.4), that is, $|f| \in \operatorname{Lip}(\alpha, L ; G)$.

Thus we have $\omega_{|f|}(x, r) \leq L r^{\alpha}$. Hence, we get

(a.4) $r\left|\underline{D}_{f}(r, x)\right| \leq c r^{\alpha}$, where $c=L \widetilde{c}$.

It is clear that (iii.4) is denoted here as (a.4). The implication (ii.4) $\Rightarrow$ (iii.4) is thus established.

If we suppose (iii.4), then an application of Lemma 20 shows that $f \in \operatorname{loc} \Lambda^{\alpha}(G)$, and since $G$ is a $L^{\alpha}$-extension domain, we conclude that $f \in \Lambda^{\alpha}(G)$. Thus (iii.4) implies (i.4).

Finally, the implication (i.4) $\Rightarrow$ (ii.4) is a clear corollary of the triangle inequality.

(A.2) Now we give another outline that (i.4) is equivalent to (ii.4).

Here, we use approach as in [15]. In particular, (a.4) implies that the condition (91) holds.

We consider two cases:

(1) $d(x) \leq 2|x-y|$;

(2) $s=|x-y| \leq d(x) / 2$.

Then we apply Lemma 33 on $G$ and $A=B(x, 2 s)$ in Case (1) and Case (2), respectively.

In more detail, if $|f| \in L^{\alpha}(G)$, then for every ball $B(x, r) \subset$ $G$, by (a.3), $r\left|\underline{D}_{f}(x)\right| \leq c r^{\alpha-1}$ and the condition (91) holds. Then Lemma 33 tells us that (92) holds, with a fixed constant, for all balls $B \subset G$ and all pairs of points $x_{1}, x_{2} \in B$. Next, we pick two points $x, \in G$ with $|x-y|<d(x, \partial G)$ and apply (92) with $G=A$, where $A=B(x ;|x-y|)$, letting $x_{1}=x$ and $x_{2}=y$. The resulting inequality

$$
|f(x)-f(y)| \leq \text { const }|x-y|^{\alpha}
$$

shows that $f \in \operatorname{loc} \Lambda^{\alpha}(G)$, and since $G$ is a $L^{\alpha}$-extension domain, we conclude that $f \in \Lambda^{\alpha}(G)$.

The implication (ii.4) $\Rightarrow$ (i.4) is thus established. The converse being trivially true.

The consideration in (A.1) shows that (i.4) and (ii.4) are equivalent with (a.4).

\section{Appendices}

\section{A. Distortion of Harmonic Maps}

Recall by $\mathbb{D}$ and $\mathbb{T}=\partial \mathbb{D}$ we denote the unit disc and the unit circle respectively, and we also use notation $z=r e^{i \theta}$. For a function $h$ we denote by $h_{r}^{\prime}, h_{x}^{\prime}$ and $h_{y}^{\prime}$ (or sometimes by $\partial_{r} h, \partial_{x} h$, and $\left.\partial_{x} h\right)$ partial derivatives with respect to $r, x$, and $y$, respectively. Let $h=f+\bar{g}$ be harmonic, where $f$ and $g$ are analytic, and every complex valued harmonic function $h$ on simply connected set $D$ is of this form. Then $\partial h=f^{\prime}$, $h_{r}^{\prime}=f_{r}^{\prime}+\overline{g_{r}^{\prime}}, f_{r}^{\prime}=f^{\prime}(z) e^{i \theta}$, and $J_{h}=\left|f^{\prime}\right|^{2}-\left|g^{\prime}\right|^{2}$. If $h$ is univalent, then $\left|g^{\prime}\right|<\left|f^{\prime}\right|$ and therefore $\left|h_{r}^{\prime}\right| \leq\left|f_{r}^{\prime}\right|+\left|g_{r}^{\prime}\right|$ and $\left|h_{r}^{\prime}\right|<2\left|f^{\prime}\right|$.

After writing this paper and discussion with some colleagues (see Remark A.11 below), the author found out that it is useful to add this section. For origins of this section see also [29].

\section{Theorem A.1. Suppose that}

(a) $h$ is an euclidean univalent harmonic mapping from an open set $D$ which contains $\overline{\mathbb{D}}$ into $\mathbb{C}$;

(b) $h(\overline{\mathbb{D}})$ is a convex set in $\mathbb{C}$;

(c) $h(\mathbb{D})$ contains a disc $B(a ; R), h(0)=a$, and $h(\mathbb{T})$ belongs to the boundary of $h(\overline{\mathbb{D}})$.

Then

(d) $\left|h_{r}^{\prime}\left(e^{i \varphi}\right)\right| \geq R / 2,0 \leq \varphi \leq 2 \pi$.

A generalization of this result to several variables has been communicated at Analysis Belgrade Seminar, cf. [32, 33].

Proposition A.2. Suppose that

$\left(\mathrm{a}^{\prime}\right) h$ is an euclidean harmonic orientation preserving univalent mapping from an open set $D$ which contains $\overline{\mathbb{D}}$ into $\mathbb{C}$;

$\left(\mathrm{b}^{\prime}\right) h(\overline{\mathbb{D}})$ is a convex set in $\mathbb{C}$;

$\left(c^{\prime}\right) h(\mathbb{D})$ contains a disc $B(a ; R)$ and $h(0)=a$.

Then

$\left(\mathrm{d}^{\prime}\right)|\partial h(z)| \geq R / 4, \quad z \in \mathbb{D}$.

By (d), we have

(e) $\left|f^{\prime}\right| \geq R / 4$ on $\mathbb{T}$.

Since $h$ is an euclidean univalent harmonic mapping, $f^{\prime} \neq$ 0 . Using (e) and applying Maximum Principle to the analytic function $f^{\prime}=\partial h$, we obtain Proposition A.2.

Proof of Theorem A.1. Without loss of generality we can suppose that $h(0)=0$. Let $0 \leq \varphi \leq 2 \pi$ be arbitrary. Since $h(\mathbb{D})$ is a bounded convex set in $\mathbb{C}$ there exists $\tau \in[0,2 \pi]$ such that harmonic function $u$, defined by $u=\operatorname{Re} H$, where $H(z)=e^{i \tau} h(z)$, has a maximum on $\overline{\mathbb{D}}$ at $e^{i \varphi}$.

Define $u_{0}(z)=u\left(e^{i \varphi}\right)-u(z), z \in \mathbb{D}$. By the mean value theorem, $(1 / 2 \pi) \int_{0}^{2 \pi} u_{0}\left(e^{i \theta}\right) d \theta=u\left(e^{i \varphi}\right)-u(0) \geq R$.

Since Poisson kernel for $\mathbb{D}$ satisfies

$$
P_{r}(\theta) \geq \frac{1-r}{1+r}
$$


using Poisson integral representation of the function $u_{0}(z)=$ $u\left(e^{i \varphi}\right)-u(z), z \in \mathbb{D}$, we obtain

$$
u\left(e^{i \varphi}\right)-u\left(r e^{i \varphi}\right) \geq \frac{1-r}{1+r}\left(u\left(e^{i \varphi}\right)-u(0)\right),
$$

and hence (d).

Now we derive a slight generalization of Proposition A.2. More precisely, we show that we can drop the hypothesis $\left(\mathrm{a}^{\prime}\right)$ and suppose weaker hypothesis $\left(\mathrm{a}^{\prime \prime}\right)$.

Proposition A.3. Suppose that $\left(a^{\prime \prime}\right) h$ is an euclidean harmonic orientation preserving univalent mapping of the unit disc onto convex domain $\Omega$. If $\Omega$ contains a disc $B(a ; R)$ and $h(0)=a$, then

$$
|\partial h(z)| \geq \frac{R}{4}, \quad z \in \mathbb{D}
$$

A proof of the proposition can be based on Proposition A.2 and the hereditary property of convex functions: (i) if an analytic function maps the unit disk univalently onto a convex domain, then it also maps each concentric subdisk onto a convex domain. It seems that we can also use the approach as in the proof of Proposition A.7, but an approximation argument for convex domain $G$, which we outline here, is interesting in itself:

(ii) approximation of convex domain $G$ with smooth convex domains.

Let $\phi$ be conformal mapping of $\mathbb{D}$ onto $G, \phi^{\prime}(0)>0, G_{n}=$ $\phi\left(r_{n} \mathbb{D}\right), r_{n}=n /(n+1), h$ is univalent mapping of the unit disc onto convex domain $\Omega$ and $D_{n}=h^{-1}\left(G_{n}\right)$.

(iii) Let $\varphi_{n}$ be conformal mapping of $\mathbb{U}$ onto $D_{n}, \varphi_{n}(0)=0$, $\varphi_{n}^{\prime}(0)>0$, and $h_{n}=h \circ \varphi_{n}$. Since $D_{n} \subset D_{n+1}$ and $\cup D_{n}=\mathbb{D}$, we can apply the Carathéodory theorem; $\varphi_{n}$ tends to $z$, uniformly on compacts, whence $\varphi_{n}^{\prime}(z) \rightarrow$ $1(n \rightarrow \infty)$. By the hereditary property $G_{n}$ is convex.

(iv) Since the boundary of $D_{n}$ is an analytic Jordan curve, the mapping $\varphi_{n}$ can be continued analytically across $\mathbb{T}$, which implies that $h_{n}$ has a harmonic extension across $\mathbb{T}$.

Thus we have the following.

(v) $h_{n}$ are harmonic on $\overline{\mathbb{D}}, G_{n}=h_{n}(\mathbb{D})$ are smooth convex domains, and $h_{n}$ tends to $h$, uniformly on compacts subset of $\mathbb{D}$.

Using (v), an application of Proposition A.2 to $h_{n}$, gives the proof.

As a corollary of Proposition A.3 we obtain (A.4).

Proposition A.4. Let $h$ be an euclidean harmonic orientation preserving $K$-qc mapping of the unit disc $\mathbb{D}$ onto convex domain $\Omega$. If $\Omega$ contains a disc $B(a ; R)$ and $h(0)=a$, then

$$
\begin{gathered}
\lambda_{h}(z) \geq \frac{1-k}{4} R, \\
\left|h\left(z_{2}\right)-h\left(z_{1}\right)\right| \geq c^{\prime}\left|z_{2}-z_{1}\right|, \quad z_{1}, z_{2} \in \mathbb{D} .
\end{gathered}
$$

(i.0) In particular, $f^{-1}$ is Lipschitz on $\Omega$.
It is worthy to note that (A.4) holds (i.e., $f^{-1}$ is Lipschitz) under assumption that $\Omega$ is convex (without any smoothness hypothesis).

Example A.5. $f(z)=(z-1)^{2}$ is univalent on $\mathbb{D}$. Since $f^{\prime}(z)=2(z-1)$ it follows that $f^{\prime}(z)$ tends 0 if $z$ tends to 1 . This example shows that we cannot drop the hypothesis that $f(\mathbb{D})$ is a convex domain in Proposition A.4.

Proof. Let $c^{\prime}=((1-k) / 4) R$. Since

$$
|\bar{D} h(z)| \leq k|D h(z)|,
$$

it follows that $\lambda_{h}(z) \geq c_{0}=((1-k) / 4) R$ and therefore $\mid h\left(z_{2}\right)-$ $h\left(z_{1}\right)\left|\geq c^{\prime}\right| z_{2}-z_{1} \mid$.

Hall, see [43, pages 66-68], proved the following.

Lemma A.6 (Hall Lemma). For all harmonic univalent mappings $f$ of the unit disk onto itself with $f(0)=0$,

$$
\left|a_{1}\right|^{2}+\left|b_{1}\right|^{2} \geq c_{0}=\frac{27}{4 \pi^{2}}
$$

where $a_{1}=D f(0), b_{1}=\bar{D} f(0)$, and $c_{0}=27 / 4 \pi^{2}=0.6839 \ldots$

Set $\tau_{0}=\sqrt{c_{0}}=3 \sqrt{3} / 2 \pi$. Now we derive a slight generalization of Proposition A.3. More precisely, we show that we can drop the hypothesis that the image of the unit disc is convex.

Proposition A.7. Let $h$ be an euclidean harmonic orientation preserving univalent mapping of the unit disc into $\mathbb{C}$ such that $f(\mathbb{D})$ contains a disc $B_{R}=B(a ; R)$ and $h(0)=a$.

(i.1) Then

$$
|\partial h(0)| \geq \frac{R}{4}
$$

(i.2) The constant $1 / 4$ in inequality (A.8) can be replaced with sharp constant $\tau_{1}=3 \sqrt{3 / 2} / 2 \pi$.

(i.3) If in addition $h$ is $K$-qc mapping and $k=(K-1) /(K+$ 1), then

$$
\lambda_{h}(0) \geq R \frac{\tau_{0}(1-k)}{\sqrt{1+k^{2}}} .
$$

Proof. Let $0<r<R$ and $V=V_{r}=h^{-1}\left(B_{r}\right)$ and let $\varphi$ be a conformal mapping of the unit disc $\mathbb{D}$ onto $V$ such that $\varphi(0)=$ 0 and let $h_{r}=h \circ \varphi$. By Schwarz lemma

$$
\left|\varphi^{\prime}(0)\right| \leq 1
$$

The function $h_{r}$ has continuous partial derivatives on $\overline{\mathbb{D}}$. Since $\partial h_{r}(0)=\partial h(0) \varphi^{\prime}(0)$, by Proposition A.3, we get $\left|\partial h_{r}(0)\right|=$ $|\partial h(0)|\left|\varphi^{\prime}(0)\right| \geq r / 4$. Hence, using (A.10) we find $|\partial h(0)| \geq$ $r / 4$ and if $r$ tends to $R$, we get (A.8).

(i2) If $r_{0}=\max \left\{|z|: z \in V_{R}\right\}$ and $q_{0}=1 / r_{0}$, then

$$
\text { (vi) } q_{0}\left|\partial h_{r}(0)\right| \leq|\partial h(0)| \text {. }
$$


Hence, by the Hall lemma, $2\left|a_{1}\right|^{2}>\left|a_{1}\right|^{2}+\left|b_{1}\right|^{2} \geq c_{0}$ and therefore

(vii) $\left|a_{1}\right| \geq \tau_{1}$, where $\tau_{1}=3 \sqrt{3 / 2} / 2 \pi$. Combining (vi) and (vii), we prove (i2).

(i3) If $h=f+\bar{g}$, where $f$ and $g$ are analytic, then $\lambda_{h}(z)=$ $\left|f^{\prime}(z)\right|-\left|g^{\prime}(z)\right|$ and $\lambda_{h}(z)=\left|f^{\prime}(z)\right|-\left|g^{\prime}(z)\right| \geq(1-$ $k)\left|f^{\prime}(z)\right|$. Set $a_{1}=D h(0)$ and $b_{1}=\bar{D} h(0)$. By the Hall sharp form $\left|a_{1}\right|^{2}+\left|b_{1}\right|^{2} \geq c_{0} R^{2}$ we get $\left|a_{1}\right|^{2}\left(1+k^{2}\right) \geq$ $c_{0} R^{2}$, then $\left|a_{1}\right| \geq R\left(\tau_{0} / \sqrt{1+k^{2}}\right)$ and therefore (A.9).

Also as a corollary of Proposition A.3 we obtain the following.

Proposition A.8 (see $[27,44])$. Let $h$ be an euclidean harmonic diffeomorphism of the unit disc onto convex domain $\Omega$. If $\Omega$ contains a disc $B(a ; R)$ and $h(0)=a$, then

$$
e(h)(z) \geq \frac{1}{16} R^{2}, \quad z \in \mathbb{D},
$$

where $e(h)(z)=|\partial h(z)|^{2}+|\bar{\partial} h(z)|^{2}$.

The following example shows that Theorem A.1 and Propositions A.2, A.3, A.4, A.7, and A.8 are not true if we omit the condition $h(0)=a$.

Example A.9. The mapping

$$
\varphi_{b}(z)=\frac{z-b}{1-\bar{b} z}, \quad|b|<1,
$$

is a conformal automorphism of the unit disc onto itself and

$$
\left|\varphi_{b}^{\prime}(z)\right|=\frac{1-|b|^{2}}{|1-\bar{b} z|^{2}}, \quad z \in \mathbb{D} .
$$

In particular $\varphi_{b}^{\prime}(0)=1-|b|^{2}$.

Heinz proved (see [45]); that if $h$ is a harmonic diffeomorphism of the unit disc onto itself such that $h(0)=0$, then

$$
e(h)(z) \geq \frac{1}{\pi^{2}}, \quad z \in \mathbb{D} .
$$

Using Proposition A.3 we can prove another Heinz theorem.

Theorem A.10 (Heinz). There exists no euclidean harmonic diffeomorphism from the unit disc $\mathbb{D}$ onto $\mathbb{C}$.

Note that this result was a key step in his proof of the Bernstein theorem for minimal surfaces in $\mathbb{R}^{3}$.

Remark A.11. Professor Kalaj turned my attention to the fact that in Proposition 12, the constant $1 / 4$ can be replaced with sharp constant $\tau_{1}=3 \sqrt{3 / 2} / 2 \pi$ which is approximately 0.584773 .
Thus Hall asserts the sharp form $\left|a_{1}\right|^{2}+\left|b_{1}\right|^{2} \geq c_{0}=$ $27 / 4 \pi^{2}$, where $c_{0}=27 / 4 \pi^{2}=0.6839 \ldots$ and therefore if $b_{1}=0$, then $\left|a_{1}\right| \geq \tau_{0}$, where $\tau_{0}=\sqrt{c_{0}}=3 \sqrt{3} / 2 \pi$.

If we combine Hall's sharp form with the Schwarz lemma for harmonic mappings, we conclude that if $a_{1}$ is real, then $3 \sqrt{3} / 2 \pi \leq a_{1} \leq 1$.

Concerning general codomains, the author, using Hall's sharp result, communicated around 1990 at Seminar University of Belgrade a proof of Corollary 16 and a version of Proposition 13; cf. [32, 33].

A.1. Characterization of Harmonic qc Mappings (See [25]). By $\chi$ we denote restriction of $h$ on $\mathbb{R}$. If $h \in H Q C_{0}(\mathbb{U})$, it is well-known that $\chi: \mathbb{R} \rightarrow \mathbb{R}$ is a homeomorphism and Re $h=P[\chi]$. Now we give characterizations of $h \in H Q C_{0}(\mathbb{Q})$ in terms of its boundary value $\chi$.

Suppose that $h$ is an orientation preserving diffeomorphism of $\mathbb{W}$ onto itself, continuous on $\mathbb{U} \cup \mathbb{R}$ such that $h(\infty)=$ $\infty$, and $\chi$ the is restriction of $h$ on $\mathbb{R}$. Recall $h \in H Q C_{0}(\mathbb{Q})$ if and only if there is analytic function $\phi: \mathbb{U} \rightarrow \Pi^{+}$such that $\phi(\mathbb{W})$ is relatively compact subset of $\Pi^{+}$and $\chi^{\prime}(x)=\operatorname{Re} \phi^{*}(x)$ a.e.

We give similar characterizations in the case of the unit disk and for smooth domains (see below).

Theorem A.12. Let $\psi$ be a continuous increasing function on $\mathbb{R}$ such that $\psi(t+2 \pi)-\psi(t)=2 \pi, \gamma(t)=e^{i \psi(t)}$ and $h=P[\gamma]$. Then $h$ is qc if and only if the following hold:

(1) ess inf $\psi^{\prime}>0$;

(2) there is analytic function $\phi: \mathbb{D} \rightarrow \Pi^{+}$such that $\phi(U)$ is relatively compact subset of $\Pi^{+}$and $\psi^{\prime}(x)=$ $\operatorname{Re} \phi^{*}\left(e^{i x}\right)$ a.e.

In the setting of this theorem we write $h=h^{\phi}$. The reader can use the above characterization and functions of the form $\phi(z)=2+M(z)$, where $M$ is an inner function, to produce examples of HQC mappings $h=h^{\phi}$ of the unit disk onto itself so the partial derivatives of $h$ have no continuous extension to certain points on the unit circle. In particular we can take $M(z)=\exp ((z+1) /(z-1))$; for the subject of this subsections cf. $[25,28]$ and references cited therein.

Remark A.13. Because of lack of space in this paper we could not consider some basic concepts related to the subject and in particular further distortion properties of qc maps as Gehring and Osgood inequality [12]. For an application of this inequality, see $[25,28]$.

\section{B. Quasi-Regular Mappings}

(A) The theory of holomorphic functions of one complex variable is the central object of study in complex analysis. It is one of the most beautiful and most useful parts of the whole mathematics.

Holomorphic functions are also sometimes referred to as analytic functions, regular functions, complex differentiable functions or conformal maps. 
This theory deals only with maps between two-dimensional spaces (Riemann surfaces).

For a function which has a domain and range in the complex plane and which preserves angles, we call a conformal map. The theory of functions of several complex variables has a different character, mainly because analytic functions of several variables are not conformal.

Conformal maps can be defined between Euclidean spaces of arbitrary dimension, but when the dimension is greater than 2, this class of maps is very small. A theorem of J. Liouville states that it consists of Mobius transformations only; relaxing the smoothness assumptions does not help, as proved by Reshetnyak. This suggests the search of a generalization of the property of conformality which would give a rich and interesting class of maps in higher dimension.

The general trend of the geometric function theory in $\mathbb{R}^{n}$ is to generalize certain aspects of the analytic functions of one complex variable. The category of mappings that one usually considers in higher dimensions is the mappings with finite distortion, thus, in particular, quasiconformal and quasiregular mappings.

For the dimensions $n=2$ and $K=1$, the class of $K$-quasiregular mappings agrees with that of the complex-analytic functions. Injective quasi-regular mappings in dimensions $n \geq 2$ are called quasiconformal. If $G$ is a domain in $\mathbb{R}^{n}$, $n \geq 2$, we say that a mapping $f: G \rightarrow \mathbb{R}^{n}$ is discrete if the preimage of a point is discrete in the domain $G$. Planar quasiregular mappings are discrete and open (a fact usually proved via Stoillow's Theorem).

Theorem A (Stoïlow's Theorem). For $n=2$ and $K \geq 1, a K$ quasi-regular mapping $f: G \rightarrow \mathbb{R}^{2}$ can be represented in the form $f=\phi \circ g$, where $g: G \rightarrow G^{\prime}$ is a $K$-quasi-conformal homeomorphism and $\phi$ is an analytic function on $G^{\prime}$.

There is no such representation in dimensions $n \geq 3$ in general, but there is representation of Stoillow's type for quasiregular mappings $f$ of the Riemann $n$-sphere $\mathbb{S}^{n}=\overline{\mathbb{R}}^{n}$, cf. [46].

Every quasiregular map $f: \mathbb{S}^{n} \rightarrow \mathbb{S}^{n}$ has a factorization $f=\phi \circ g$, where $g: \mathbb{S}^{n} \rightarrow \mathbb{S}^{n}$ is quasiconformal and $\phi:$ $\mathbb{S}^{n} \rightarrow \mathbb{S}^{n}$ is uniformly quasiregular.

Gehring-Lehto Lemma: let $f$ be a complex, continuous, and open mapping of a plane domain $\Omega$ which has finite partial derivatives a.e. in $\Omega$. Then $f$ is differentiable a.e. in $\Omega$.

Let $U$ be an open set in $\mathbb{R}^{n}$, and let $f: U \rightarrow \mathbb{R}^{m}$ be a mapping. Set

$$
L(x, f)=\lim \sup \frac{|f(x+h)-f(x)|}{h} .
$$

If $f$ is differentiable at $x$, then $L(x, f)=\left|f^{\prime}(x)\right|$. The theorem of Rademacher-Stepanov states that if $L(x, f)<\infty$ a.e., then $f$ is differentiable a.e.

Note that Gehring-Lehto Lemma is used in dimension $n=2$ and Rademacher-Stepanov theorem to show the following.

For all dimensions, $n \geq 2$, a quasiconformal mapping $f$ : $G \rightarrow \mathbb{R}^{n}$, where $G$ is a domain in $\mathbb{R}^{n}$, is differentiable a.e. in G.
Therefore the set $S_{f}$ of those points where it is not differentiable has Lebesgue measure zero. Of course, $S_{f}$ may be nonempty in general and the behaviour of the mapping may be very interesting at the points of this set. Thus, there is a substantial difference between the two cases $K>1$ and $K=$ 1 . This indicates that the higher dimensions theory of quasiregular mappings is essentially different from the theory in the complex plane. There are several reasons for this:

(a) there are neither general representation theorems of Stoillow's type nor counterparts of power series expansions in higher dimensions;

(b) the usual methods of function theory based on Cauchy's Formula, Morera's Theorem, Residue Theorem, The Residue Calculus and Consequences, Laurent Series, Schwarz's Lemma, Automorphisms of the Unit Disc, Riemann Mapping Theorem, and so forth, are not applicable in the higher-dimensional theory;

(c) in the plane case the class of conformal mappings is very rich, while in higher dimensions it is very small (J. Liouville proved that for $n \geq 3$ and $K=1$, sufficiently smooth quasiconformal mappings are restrictions of Möbius transformations);

(d) for dimensions $n \geq 3$ the branch set (i.e., the set of those points at which the mapping fails to be a local homeomorphism) is more complicated than in the two-dimensional case; for instance, it does not contain isolated points.

Injective quasiregular maps are called quasiconformal. Using the interaction between different coordinate systems, for example, spherical coordinates $(\rho, \theta, \phi) \in R_{+} \times[0,2 \pi) \times$ $[0, \pi]$ and cylindrical coordinates $(r, \theta, t)$, one can construct certain qc maps.

Define $f$ by $(\rho, \theta, \phi) \mapsto(\phi, \theta, \ln \rho)$. Then $f$ maps the cone $C\left(\phi_{0}\right)=\left\{(\rho, \theta, \phi): 0 \leq \phi<\phi_{0}\right\}$ for $0<\phi_{0} \leq \pi$ onto the infinite cylinder $\left\{(r, \theta, t): 0 \leq r<\phi_{0}\right\}$. We leave it to the reader as an exercise to check that the linear distortion $H$ depends only on $\phi$ and that $H(\rho, \theta, \phi)=\phi / \sin \phi \leq \phi_{0} / \sin \phi_{0}$.

For $\phi_{0}=\pi / 2$ we obtain a qc map of the half-space onto the cylinder with the linear distortion bounded by $\pi / 2$.

Since the half space and ball are conformally equivalent, we find that there is a qc map of the unit ball onto the infinite cylinder with the linear distortion bounded by $\pi / 2$.

A simple example of noninjective quasiregular map $f$ is given in cylindrical coordinates in 3-space by the formula $(r, \theta, z) \mapsto(r, 2 \theta, z)$. This map is two-to-one and it is quasiregular on any bounded domain in $\mathbb{R}^{3}$ whose closure does not intersect the $z$-axis. The Jacobian $J(f)$ is different from 0 except on the $z$-axis, and it is smooth everywhere except on the $z$-axis.

Set $S_{+}=\left\{(x, y, z): x^{2}+y^{2}+z^{2}=1, z \geq 0\right\}$ and $\bar{H}^{3}=$ $\{(x, y, z): z \geq 0\}$. The Zorich map $Z: \mathbb{R}^{3} \rightarrow \mathbb{R}^{3} \backslash\{0\}$ is a quasiregular analogue of the exponential function. It can be defined as follows.

(1) Choose a bi-Lipschitz map $h:[-\pi / 2, \pi / 2]^{2} \rightarrow S_{+}$. 
(2) Define $Z:[-\pi / 2, \pi / 2]^{2} \times R \rightarrow H^{3}$ by $Z(x, y, z)=$ $e^{z} h(x, y)$.

(3) Extend $Z$ to all of $\mathbb{R}^{3}$ by repeatedly reflecting in planes.

Quasiregular maps of $\mathbb{R}^{n}$ which generalize the sine and cosine functions have been constructed by Drasin, by Mayer, and by Bergweiler and Eremenko, see in Fletcher and Nicks paper [47].

(B) There are some new phenomena concerning quasiregular maps which are local homeomorphisms in dimensions $n \geq 3$. A remarkable fact is that all smooth quasiregular maps are local homeomorphisms. Even more remarkable is the following result of Zorich [48].

Theorem B.1. Every quasiregular local homeomorphism $\mathbb{R}^{n} \rightarrow \mathbb{R}^{n}$, where $n \geq 3$, is a homeomorphism.

The result was conjectured by M. A. Lavrentev in 1938. The exponential function exp shows that there is no such result for $n=2$. Zorich's theorem was generalized by Martio et al., cf. [49] (for a proof see also, e.g., [35, Chapter III, Section $3])$.

Theorem B.2. There is a number $r=r(n, K)$ (which one calls the injectivity radius) such that every $K$-quasiregular local homeomorphism $g: \mathbb{B} \rightarrow \mathbb{R}^{n}$ of the unit ball $\mathbb{B} \subset R^{n}$ is actually homeomorphic on $B(0 ; r)$.

An immediate corollary of this is Zorich's result.

This explains why in the definition of quasiregular maps it is not reasonable to restrict oneself to smooth maps: all smooth quasiregular maps of $\mathbb{R}^{n}$ to itself are quasiconformal.

In each dimension $n \geq 3$ there is a positive number $\epsilon_{n}$ such that every nonconstant quasiregular mapping $f: D \rightarrow$ $\mathbb{R}^{n}$ whose distortion function satisfies $K_{\alpha, \beta}(x, f) \leq 1+\epsilon_{n}$ for some $1 \leq \alpha, \beta \leq n-1$ is locally injective.

(C) Despite the differences between the two theories described in parts (A) and (B), many theorems about geometric properties of holomorphic functions of one complex variable have been extended to quasiregular maps. These extensions are usually highly nontrivial.

(e) In a pioneering series of papers, Reshetnyak proved in 1966-1969 that these mappings share the fundamental topological properties of complex-analytic functions: nonconstant quasi-regular mappings are discrete, open, and sense-preserving, cf. [50]. Here we state only the following.

Open Mapping Theorem. If $D$ is open in $\mathbb{R}^{n}$ and $f$ is a nonconstant qr function from $D$ to $\mathbb{R}^{n}$, we have that $f(D)$ is open set. (Note that this does not hold for real analytic functions).

An immediate consequence of the open mapping theorem is the maximum modulus principle. It states that if $f$ is $\mathrm{qr}$ in a domain $D$ and $|f|$ achieves its maximum on $D$, then $f$ is constant. This is clear. Namely, if $a \in D$ then the open mapping theorem says that $f(a)$ is an interior point of $f(D)$ and hence there is a point in $D$ with larger modulus. (f) Reshetnyak also proved important convergence theorems for these mappings and several analytic properties: they preserve sets of zero Lebesgue-measure, are differentiable almost everywhere, and are Hölder continuous. The Reshetnyak theory (which uses the phrase mapping with bounded distortion for "quasiregular mapping") makes use of Sobolev spaces, potential theory, partial differential equations, calculus of variations, and differential geometry. Those mappings solve important first-order systems of PDEs analogous in many respects to the Cauchy-Riemann equation. The solutions of these systems can be viewed as "absolute" minimizers of certain energy functionals.

(g) We have mentioned that all pure topological results about analytic functions (such as the Maximum Modulus Principle and Rouché's theorem) extend to quasiregular maps. Perhaps the most famous result of this sort is the extension of Picard's theorem which is due to Rickman, cf. [35]: set.

A $K$-quasiregular map $\mathbb{R}^{n} \rightarrow \mathbb{R}^{n}$ can omit at most a finite

When $n=2$, this omitted set can contain at most two points (this is a simple extension of Picard's theorem). But when $n>2$, the omitted set can contain more than two points, and its cardinality can be estimated from above in terms of $n$ and $K$. There is an integer $q=q(n, K)$ such that every $K$-qr mapping $f: \mathbb{R}^{n} \rightarrow \overline{\mathbb{R}}^{n} \backslash\left\{a_{1}, a_{2}, \ldots, a_{q}\right\}$, where $a_{j}$ are disjoint, is constant. It was conjectured for a while that $q(n, K)=2$. Rickman gave a highly nontrivial example to show that it is not the case: for every positive integer $p$ there exists a nonconstant $K$-qr mapping $f: \mathbb{R}^{3} \rightarrow \mathbb{R}^{3}$ omitting $p$ points.

(h) It turns out that these mappings have many properties similar to those of plane quasiconformal mappings. On the other hand, there are also striking differences. Probably the most important of these is that there exists no analogue of the Riemann mapping theorem when $n>2$. This fact gives rise to the following two problems. Given a domain $D$ in Euclidean $n$-space, does there exist a quasiconformal homeomorphism $f$ of $D$ onto the $n$-dimensional unit ball $\mathbb{B}^{n}$ ? Next, if such a homeomorphism $f$ exists, how small can the dilatation of $f$ be?

Complete answers to these questions are known when $n=$ 2. For a plane domain $D$ can be mapped quasiconformally onto the unit disk $B$ if and only if $D$ is simply connected and has at least two boundary points. The Riemann mapping theorem then shows that if $D$ satisfies these conditions, there exists a conformal homeomorphism $f$ of $D$ onto $\mathbb{D}$. The situation is very much more complicated in higher dimensions, and the Gehring-Väisälä paper [51] is devoted to the study of these two questions in the case where $n=3$.

(D) We close this subsection with short review of Dyakonov's approach [15].

The main result of Dyakonov's papers [6] (published in Acta Math.) is as follows. 
Theorem B. Lipschitz-type properties are inherited from its modulus by analytic functions.

A simple proof of this result was also published in Acta Math. by Pavlović, cf. [52].

(D1) In this item we shortly discuss the Lipschitz-type properties for harmonic functions. The set of harmonic functions on a given open set $U$ can be seen as the kernel of the Laplace operator $\Delta$ and is therefore a vector space over $\mathbb{R}$ : sums, differences, and scalar multiples of harmonic functions are again harmonic. In several ways, the harmonic functions are real analogues to holomorphic functions. All harmonic functions are real analytic; that is, they can be locally expressed as power series, they satisfy the mean value theorem, there is Liouville's type theorem for them, and so forth.

Harmonic quasiregular (briefly, hqr) mappings in the plane were studied first by Martio in [53]; for a review of this subject and further results, see $[25,54]$ and the references cited there. The subject has grown to include study of hqr maps in higher dimensions, which can be considered as a natural generalization of analytic function in plane and good candidate for a generalization of Theorem B.

For example, Chen et al. [19] and the author [55] have shown that Lipschitz-type properties are inherited from its modulo for $K$-quasiregular and harmonic mappings in planar case. These classes include analytic functions in planar case, so this result is a generalization of Theorem A.

(D2) Quasiregular mappings. Dyakonov [15] made further important step and roughly speaking showed that Lipschitz-type properties are inherited from its modulus by qc mappings (see two next results).

Theorem B.3 (Theorem Dy, see [15, Theorem 4]). Let $0<$ $\alpha \leq 1, K \geq 1$, and $n \geq 2$. Suppose $G$ is a $\Lambda^{\alpha}$-extension domain in $\mathbb{R}^{n}$ and $f$ is a $K$-quasiconformal mapping of $G$ onto $f(G) \subset \mathbb{R}^{n}$. Then the following conditions are equivalent:

(i.5) $f \in \Lambda^{\alpha}(G)$;

(ii.5) $|f| \in \Lambda^{\alpha}(G)$.

If, in addition, $G$ is a uniform domain and if $\alpha \leq \alpha_{0}=\alpha_{K}^{n}=$ $K^{1 /(1-n)}$, then (i.5) and (ii.5) are equivalent to

(iii.5) $|f| \in \Lambda^{\alpha}(G, \partial G)$.

An example in Section 2 [15] shows that the assumption $\alpha \leq \alpha_{0}=\alpha_{K}^{n}=K^{1 /(1-n)}$ cannot be dropped. For $n \geq 3$, we have the following generalization-but also a consequence of Theorem B.3 dealing with quasiregular mappings that are local homeomorphisms (i.e., have no branching points).

Theorem B.4 (see [15, Proposition 3]). Let $0<\alpha \leq 1, K \geq 1$, and $n \geq 3$. Suppose $G$ is a $\Lambda^{\alpha}$-extension domain in $\mathbb{R}^{n}$ and $f$ is a K-quasiregular locally injective mapping of $G$ onto $f(G) \subset$ $\mathbb{R}^{n}$. Then $f \in \Lambda^{\alpha}(G)$ if and only if $|f| \in \Lambda^{\alpha}(G)$.
We remark that a quasiregular mapping $f$ in dimension $n \geq 3$ will be locally injective (or, equivalently, locally homeomorphic) if the dilatation $K(f)$ is sufficiently close to 1 . For more sophisticated local injectivity criteria, see the literature cited in $[10,15]$.

Here we only outline how to reduce the proof of Theorem B.4 to qc case.

It is known that, by Theorem B.2, for given $n \geq 3$ and $K \geq 1$, there is a number $r=r(n, K)$ such that every $K$ quasiregular local homeomorphism $g: \mathbb{B} \rightarrow R^{n}$ of the unit ball $\mathbb{B} \subset \mathbb{R}^{n}$ is actually homeomorphic on $B(0 ; r)$. Applying this to the mappings $g_{x}(w)=f(x+d(x) w), w \in \mathbb{B}$, where $x \in$ $G$ and $d(x)=d(x, \partial G)$, we see that $f$ is homeomorphic (and hence quasiconformal) on each ball $B_{r}(x):=B(0 ; r d(x))$. The constant $r=r(n, K) \in(0,1)$, coming from the preceding statement, depends on $n$ and $K=K(f)$, but not on $x$.

Note also the following.

(i0) Define $m_{f}(x, r)=\min \left\{\left|f\left(x^{\prime}\right)-f(x)\right|:\left|x^{\prime}-x\right|=r\right\}$ and $M_{f}(x, r)=\max \left\{\left|f\left(x^{\prime}\right)-f(x)\right|:\left|x^{\prime}-x\right|=r\right\}$. We can express the distortion property of qc mappings in the following useful form.

Proposition A. Suppose that $G$ is a domain in $\mathbb{R}^{n}$ and $f$ : $G \rightarrow \mathbb{R}^{n}$ is K-quasiconformal and $G^{\prime}=f(G)$. There is a constant $c$ such that $M_{f}(x, r) \leq c m_{f}(x, r)$ for $x \in G$ and $r=$ $d(x) / 2$. This form shows in an explicit way that the maximal dilatation of a qc mapping is controlled by minimal and it is convenient for some applications. For example, Proposition A also yields a simple proof of Theorem B.3.

Recall the following. (i1) Roughly speaking, quasiconformal and quasiregular mappings in $\mathbb{R}^{n}, n \geq 3$, are natural generalizations of conformal and analytic functions of one complex variable.

(i2) Dyakonov's proof of Theorem 6.4 in [15] (as we have indicated above) is reduced to quasiconformal case, but it seems likely that he wanted to consider whether the theorem holds more generally for quasiregular mappings.

(i3) Quasiregular mappings are much more general than quasiconformal mappings and in particular analytic functions.

(E) Taking into account the above discussion it is natural to explore the following research problem.

Question A. Is it possible to drop local homeomorphism hypothesis in Theorem B.4?

It seems that using the approach from [15], we cannot solve this problem and that we need new techniques.

(i4) However, we establish the second version of Koebe theorem for $K$-quasiregular functions, Theorem 39, and the characterization of Lipschitz-type spaces for quasiregular mappings by the average Jacobian, Theorem 40. Using these theorems, we give a positive solution to Question A.

\section{Conflict of Interests}

The author declares that there is no conflict of interests regarding the publication of this paper. 


\section{Acknowledgments}

The author is grateful to the anonymous referees for a number of helpful suggestions and in particular to the Editor in Chief Professor Cirić for patiently reading through the whole text at several stages of its revision. This research was partially supported by MNTRS, Serbia, Grant no. 174032.

\section{References}

[1] K. Astala and F. W. Gehring, "Injectivity, the BMO norm and the universal Teichmüller space," Journal d’Analyse Mathématique, vol. 46, pp. 16-57, 1986.

[2] F. W. Gehring and O. Martio, "Lipschitz classes and quasiconformal mappings," Annales Academioe Scientiarum Fennicœ. Series A I. Mathematica, vol. 10, pp. 203-219, 1985.

[3] C. A. Nolder, "A quasiregular analogue of a theorem of Hardy and Littlewood," Transactions of the American Mathematical Society, vol. 331, no. 1, pp. 215-226, 1992.

[4] K. Astala and F. W. Gehring, "Quasiconformal analogues of theorems of Koebe and Hardy-Littlewood," The Michigan Mathematical Journal, vol. 32, no. 1, pp. 99-107, 1985.

[5] K. Kim, "Hardy-Littlewood property with the inner length metric," Korean Mathematical Society, vol. 19, no. 1, pp. 53-62, 2004.

[6] K. M. Dyakonov, "Equivalent norms on Lipschitz-type spaces of holomorphic functions," Acta Mathematica, vol. 178, no. 2, pp. 143-167, 1997.

[7] A. Abaob, M. Arsenović, M. Mateljević, and A. Shkheam, "Moduli of continuity of harmonic quasiregular mappings on bounded domains," Annales Academice Scientiarum Fennica, vol. 38, no. 2, pp. 839-847, 2013.

[8] M. Mateljević and M. Vuorinen, "On harmonic quasiconformal quasi-isometries," Journal of Inequalities and Applications, vol. 2010, Article ID 178732, 2010.

[9] J. Qiao and X. Wang, "Lipschitz-type spaces of pluriharmonic mappings," Filomat, vol. 27, no. 4, pp. 693-702, 2013.

[10] T. Iwaniec and G. Martin, Geometric Function Theory and Nonlinear Analysis, Syraccuse, Aucland, New Zealand, 2000.

[11] M. Vuorinen, Conformal Geometry and Quasiregular Mappings, vol. 1319 of Lecture Notes in Mathematics, Springer, Berlin, Germany, 1988.

[12] F. W. Gehring and B. G. Osgood, "Uniform domains and the quasihyperbolic metric," Journal d'Analyse Mathématique, vol. 36, pp. 50-74, 1979.

[13] M. Arsenović, V. Manojlović, and M. Mateljević, "Lipschitztype spaces and harmonic mappings in the space," Annales Academice Scientiarum Fennice. Mathematica, vol. 35, no. 2, pp. 379-387, 2010.

[14] Sh. Chen, M. Mateljević, S. Ponnusamy, and X. Wang, "Lipschitz type spaces and Landau-Bloch type theorems for harmonic functions and solutions to Poisson equations," to appear.

[15] K. M. Dyakonov, "Holomorphic functions and quasiconformal mappings with smooth moduli," Advances in Mathematics, vol. 187, no. 1, pp. 146-172, 2004.

[16] A. Hinkkanen, "Modulus of continuity of harmonic functions," Journal d'Analyse Mathématique, vol. 51, pp. 1-29, 1988.

[17] V. Lappalainen, " $\mathrm{Lip}_{h}$-extension domains," Annales Academice Scientiarum Fennica. Series A I. Mathematica Dissertationes, no. 56, p. 52, 1985.
[18] O. Martio and R. Näkki, "Boundary Hölder continuity and quasiconformal mappings," Journal of the London Mathematical Society. Second Series, vol. 44, no. 2, pp. 339-350, 1991.

[19] S. Chen, S. Ponnusamy, and X. Wang, "On planar harmonic Lipschitz and planar harmonic Hardy classes," Annales Academice Scientiarum Fennicee. Mathematica, vol. 36, no. 2, pp. 567-576, 2011.

[20] Sh. Chen, S. Ponnusamy, and X. Wang, "Equivalent moduli of continuity, Bloch's theorem for pluriharmonic mappings in $B^{n}$," Proceedings of Indian Academy of Sciences-Mathematical Sciences, vol. 122, no. 4, pp. 583-595, 2012.

[21] Sh. Chen, S. Ponnusamy, M. Vuorinen, and X. Wang, "Lipschitz spaces and bounded mean oscillation of harmonic mappings," Bulletin of the Australian Mathematical Society, vol. 88, no. 1, pp. 143-157, 2013.

[22] S. Chen, S. Ponnusamy, and A. Rasila, "Coefficient estimates, Landau's theorem and Lipschitz-type spaces on planar harmonic mappings," Journal of the Australian Mathematical Society, vol. 96, no. 2, pp. 198-215, 2014.

[23] F. W. Gehring, "Rings and quasiconformal mappings in space," Transactions of the American Mathematical Society, vol. 103, pp. 353-393, 1962.

[24] J. Väisälä, Lectures on n-Dimensional Quasiconformal Mappings, Springer, New York, NY, USA, 1971.

[25] M. Mateljević, "Quasiconformality of harmonic mappings between Jordan domains," Filomat, vol. 26, no. 3, pp. 479-510, 2012.

[26] D. Kalaj and M. S. Mateljević, "Harmonic quasiconformal selfmappings and Möbius transformations of the unit ball," Pacific Journal of Mathematics, vol. 247, no. 2, pp. 389-406, 2010.

[27] D. Kalaj, "On harmonic diffeomorphisms of the unit disc onto a convex domain," Complex Variables, vol. 48, no. 2, pp. 175-187, 2003.

[28] M. Mateljević, Topics in Conformal, Quasiconformal and Harmonic Maps, Zavod za Udzbenike, Belgrade, Serbia, 2012.

[29] M. Mateljević, "Distortion of harmonic functions and harmonic quasiconformal quasi-isometry," Revue Roumaine de Mathématiques Pures et Appliquées, vol. 51, no. 5-6, pp. 711-722, 2006.

[30] M. Knežević and M. Mateljević, "On the quasi-isometries of harmonic quasiconformal mappings," Journal of Mathematical Analysis and Applications, vol. 334, no. 1, pp. 404-413, 2007.

[31] V. Manojlović, "Bi-Lipschicity of quasiconformal harmonic mappings in the plane," Filomat, vol. 23, no. 1, pp. 85-89, 2009.

[32] M. Mateljević, Communications at Analysis Seminar, University of Belgrade, 2012.

[33] M. Mateljević, "Quasiconformal and Quasiregular harmonic mappings and Applications," Annales Academice Scientiarum Fennicee Mathematica, vol. 32, pp. 301-315, 2007.

[34] I. Anić, V. Marković, and M. Mateljević, "Uniformly bounded maximal $\varphi$-disks, Bers space and harmonic maps," Proceedings of the American Mathematical Society, vol. 128, no. 10, pp. 29472956, 2000.

[35] S. Rickman, Quasiregular Mappings, vol. 26, Springer, Berlin, Germany, 1993.

[36] O. Lehto and K. I. Virtanen, Quasiconformal Mappings in the Plane, Springer, New York, NY, USA, 2nd edition, 1973.

[37] D. Gilbarg and N. S. Trudinger, Elliptic Partial Differential Equations of Second Order, vol. 224 of Grundlehren der Mathematischen Wissenschaften [Fundamental Principles of Mathematical Sciences], Springer, Berlin, Germany, 2nd edition, 1983. 
[38] B. Burgeth, "A Schwarz lemma for harmonic and hyperbolicharmonic functions in higher dimensions," Manuscripta Mathematica, vol. 77, no. 2-3, pp. 283-291, 1992.

[39] S. Axler, P. Bourdon, and W. Ramey, Harmonic Function Theory, vol. 137 of Graduate Texts in Mathematics, Springer, New York, NY, USA, 1992.

[40] W. Rudin, Function Theory in the Unit Ball of $C^{n}$, Springer, Berlin, Germany, 1980.

[41] R. Näkki and B. Palka, "Lipschitz conditions and quasiconformal mappings," Indiana University Mathematics Journal, vol. 31, no. 3, pp. 377-401, 1982.

[42] M. Mateljević, "Versions of Koebe $1 / 4$ theorem for analytic and quasiregular harmonic functions and applications," Institut Mathématique. Publications. Nouvelle Série, vol. 84, pp. 61-72, 2008.

[43] P. Duren, Harmonic Mappings in the Plane, vol. 156 of Cambridge Tracts in Mathematics, Cambridge University Press, Cambridge, UK, 2004.

[44] D. Kalaj, Harmonic functions and quasiconformal mappings [M.S. thesis], Harmonijske Funkcije i Kvazikonformna Preslikavanja, Belgrade, Serbia, 1998.

[45] E. Heinz, "On one-to-one harmonic mappings," Pacific Journal of Mathematics, vol. 9, no. 1, pp. 101-105, 1959.

[46] G. Martin and K. Peltonen, "Stoillow factorization for quasiregular mappings in all dimensions," Proceedings of the American Mathematical Society, vol. 138, no. 1, pp. 147-151, 2010.

[47] A. N. Fletcher and D. A. Nicks, "Iteration of quasiregular tangent functions in three dimensions," Conformal Geometry and Dynamics, vol. 16, pp. 1-21, 2012.

[48] V. A. Zorich, "The global homeomorphism theorem for space quasiconformal mappings, its development and related open problems," in Quasiconformal Space Mappings, vol. 1508 of Lecture Notes in Mathematics, pp. 132-148, Springer, Berlin, Germany, 1992.

[49] O. Martio, S. Rickman, and J. Väisälä, "Topological and metric properties of quasiregular mappings," Annales Academioe Scientiarum Fennicae Mathematica, vol. 488, pp. 1-31, 1971.

[50] Yu. G. Reshetnyak, "Space mappings with bounded distortion," Siberian Mathematical Journal, vol. 8, no. 3, pp. 466-487, 1967.

[51] F. W. Gehring and J. Väisälä, "The coefficients of quasiconformality of domains in space," Acta Mathematica, vol. 114, pp. 170, 1965.

[52] M. Pavlović, “On Dyakonov's paper 'Equivalent norms on lipschitz-type spaces of holomorphic functions," Acta Mathematica, vol. 183, no. 1, pp. 141-143, 1999.

[53] O. Martio, “On harmonic quasiconformal mappings," Annales Academioe Scientiarum Fennica Mathematica, vol. 425, pp. 3$10,1968$.

[54] M. Arsenović, V. Kojić, and M. Mateljević, "On Lipschitz continuity of harmonic quasiregular maps on the unit ball in $R^{n}$," Annales Academice Scientiarum Fennicoe. Mathematica, vol. 33, no. 1, pp. 315-318, 2008.

[55] M. Mateljević, "A version of Bloch's theorem for quasiregular harmonic mappings," Revue Roumaine de Mathématiques Pures et Appliquées, vol. 47, no. 5-6, pp. 705-707, 2002. 


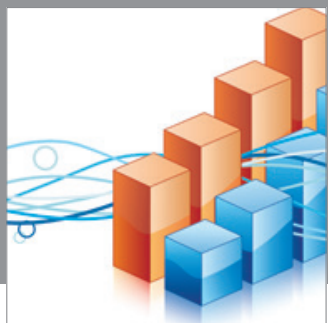

Advances in

Operations Research

mansans

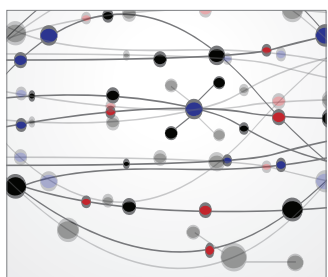

The Scientific World Journal
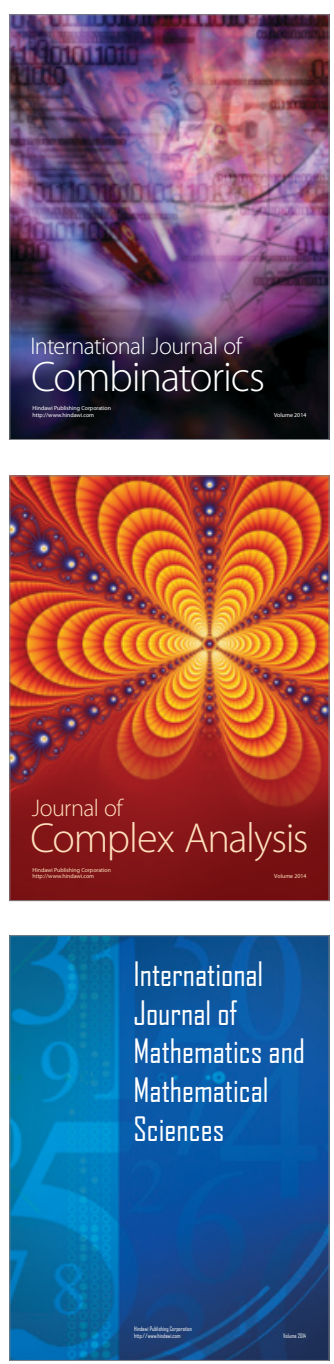
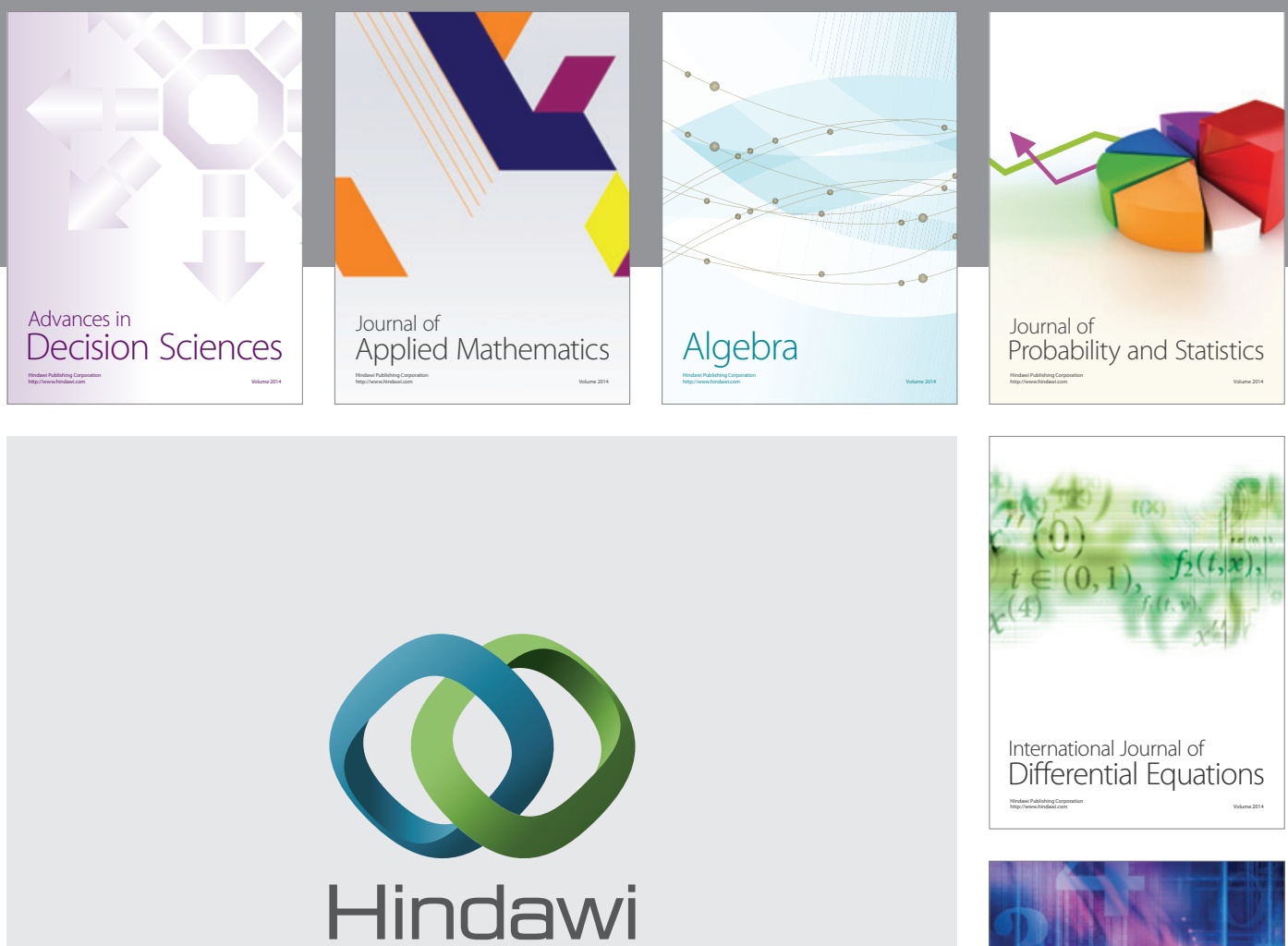

Submit your manuscripts at http://www.hindawi.com
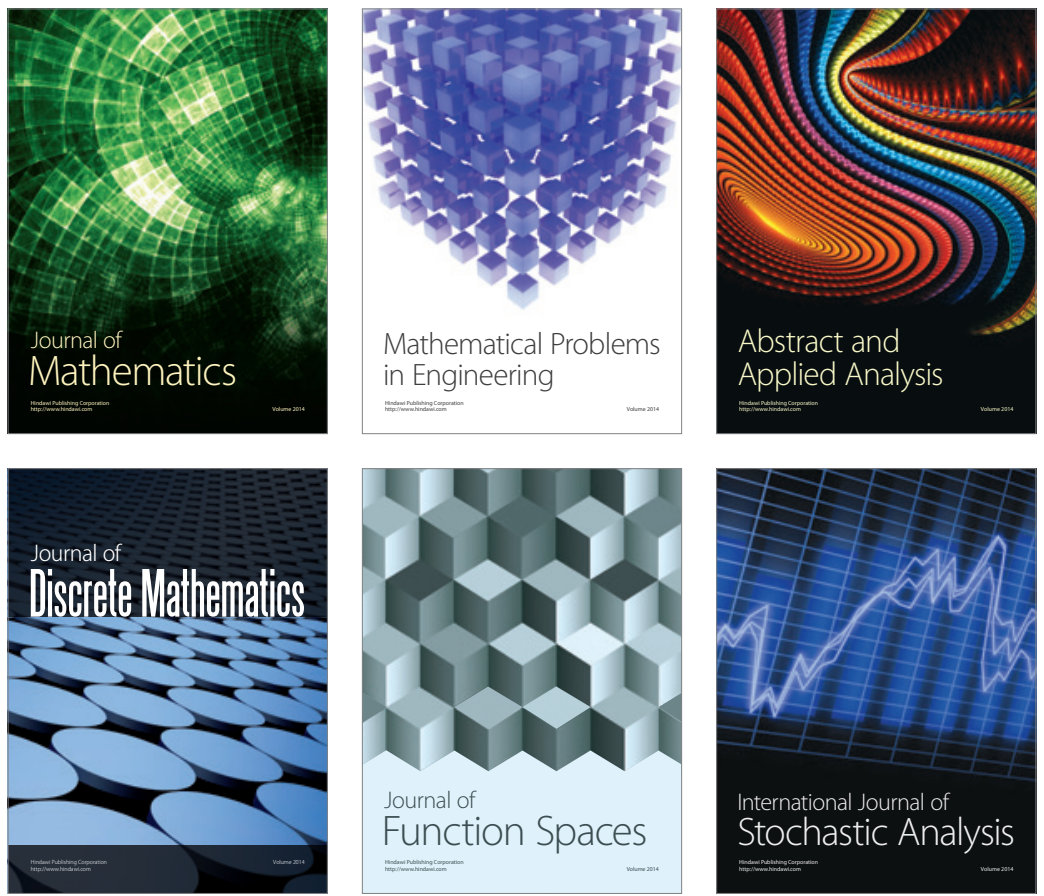

Journal of

Function Spaces

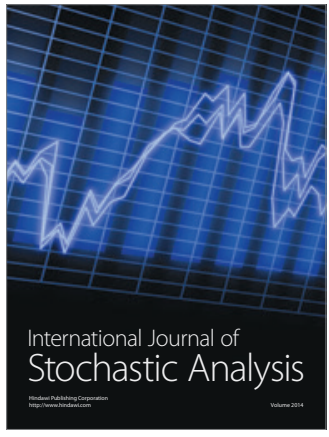

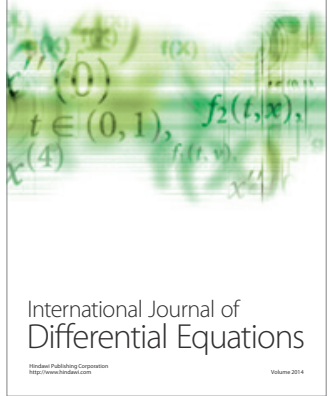
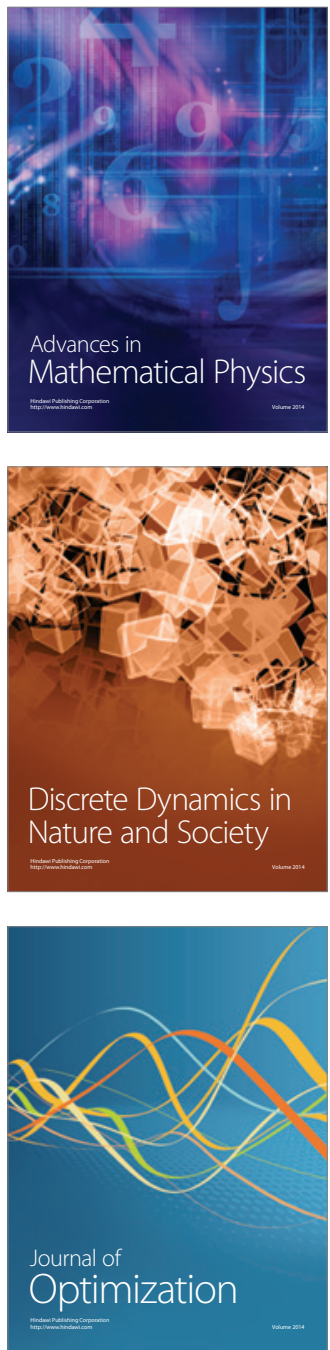